\title{
AN INTRODUCTION TO PROBABILISTIC METHODS WITH APPLICATIONS
}

\author{
Pierre Del Moral ${ }^{1}$ and Nicolas G. Hadjiconstantinou ${ }^{2}$
}

\begin{abstract}
This special volume of the ESAIM Journal, Mathematical Modelling and Numerical Analysis, contains a collection of articles on probabilistic interpretations of some classes of nonlinear integrodifferential equations. The selected contributions deal with a wide range of topics in applied probability theory and stochastic analysis, with applications in a variety of scientific disciplines, including physics, biology, fluid mechanics, molecular chemistry, financial mathematics and bayesian statistics. In this preface, we provide a brief presentation of the main contributions presented in this special volume. We have also included an introduction to classic probabilistic methods and a presentation of the more recent particle methods, with a synthetic picture of their mathematical foundations and their range of applications.
\end{abstract}

Mathematics Subject Classification. 65M75, 68Q87, 60H35, 35Q68, 37N10, 35Q35, 35Q20.

Received January 2nd, 2009.

Published online August 26, 2010.

\section{INTRODUCTION}

Probabilistic modeling and stochastic analysis can be found in virtually all scientific fields involving quantitative analysis, with applications ranging from component lifetime prediction under cyclic loading to quantum field theory.

A volume representing all application fields would simply be an impossible undertaking but undoubtedly instructive. With this volume we attempt something much less ambitious, but hopefully still useful, namely the collection of a series of representative articles in the general area of probabilistic methods. Our objective is to bring a variety of communities using similar tools and methods closer together, draw attention to the beauty and importance of these methods, as well as highlight the challenges in the field to the wider scientific community.

A rather large part of this special volume is dedicated to mean field particle models and related branching and interacting particle systems. These stochastic techniques can be thought of in various ways: From the physical view point, they can be seen as microscopic particle interpretations of physical non linear measure

Keywords and phrases. Fokker-Planck equations, Vlasov diffusion models, fluid-Lagrangian-velocities model, Boltzmann collision models, interacting jump processes, adaptive biasing force model, molecular dynamics, ground state energies, hidden Markov chain problems, Feynman-Kac semigroups, Dirichlet problems with boundary conditions, Poisson Boltzmann equations, mean field stochastic particle models, stochastic analysis, functional contraction inequalities, uniform propagation of chaos properties w.r.t. the time parameter.

1 Centre INRIA Bordeaux et Sud-Ouest \& Institut de Mathématiques de Bordeaux, Université de Bordeaux I, 351 cours de la Libération, 33405 Talence Cedex, France. Pierre.Del-Moral@inria.fr

2 Massachusetts Institute of Technology, 77 Massachusetts Avenue, Cambridge MA 02139-4307, USA. ngh@mit.edu 
valued equations. From the pure mathematical point of view, they can also be seen as a sophisticated stochastic linearizations of non linear evolution semigroups. From the statistical and the numerical point of view, these stochastic particle models are commonly used as powerful simulation techniques for sampling and computing complex probability distributions.

These interacting particle methods are one of the most active contact points between applied probability, Bayesian statistics, theoretical chemistry, quantum physics, and engineering sciences, including rare event analysis and advanced signal processing. In this volume nine selected articles are dedicated to this subject. A brief description of these studies is given below.

The article by Jourdain et al. [21] is concerned with the existence, the uniqueness and the convergence of a particle approximation for the adaptive biasing force process. The central idea of these adaptive methods is to modify the potential function of a Boltzmann-Gibbs measure so that to remove the metastable features of the corresponding Lagrangian diffusion sampling process.

The article by Bolley et al. [6] is concerned with the propagation of chaos properties and the long time behavior of a kinetic McKean-Vlasov-Fokker-Planck model related to quadratic like interaction potential and drift forces. The authors provide explicit geometric speeds of convergence towards equilibrium in Wasserstein distance.

The following two articles have applications in meteorology and forecasting problems: the article by Bernardin et al. [4] proposes a novel stochastic Lagrangian method for downscaling problems in meteorology. The central idea in this work is to compute small scale phenomena in atmospheric models without using mesh refinement. This is achieved by moving the particle simulators according to the Stochastic Lagrangian model, adapted from methods proposed by Pope. The second contribution [2], by Baehr, is concerned with nonlinear filtering of random field signals using path observation models. The author introduces a new filtering model involving partially observed mean field type signal processes. The signal stochastic model can be seen as the discrete generation version of the fluid-Lagrangian-velocities model introduced by Pope. The estimation problem of partially observed atmospheric turbulent velocities is solved using interacting particle filters and genealogical tree based smoothing techniques. In this sense, the resulting filtering process can be seen as a stochastic closure technique applied to the fluid equations developed by Pope.

The article by Del Moral et al. [16] is concerned with an interacting particle approximation of FeynmanKac semigroups in path space, with applications in numerical physics, Bayesian statistics, and advanced signal processing. These new stochastic models are based on a backward Markovian representation combined with a traditional mean field particle interpretation of the flow of the final time marginals. In contrast to more traditional genealogical tree based models, these stochastic particle algorithms can be used to compute normalized additive functionals "on-the-fly" as well as their limiting occupation measures with a given precision degree that does not depend on the final time horizon.

The following two articles make contributions to stochastic algorithms for molecular dynamics: The article by Rousset [37] is concerned with probabilistic interpretations of the shape derivative of Dirichlet groundstate energy w.r.t. domain perturbations. The author also discusses a nodal type particle approximation method. The article by Champagnat et al. [7] analyzes the random walk on spheres algorithm for the numerical solution of the Poisson-Boltzmann equation with discontinuous divergence operators. This stochastic algorithm can be interpreted as a particle absorption model with a free evolution on the biggest spheres included in some domain.

The article by Muscato et al. [33] studies the numerical error in Monte Carlo particle methods modeling carrier transport in semiconductors; both errors due to finite timestep and number of particles is examined. Due to the Monte Carlo nature of the algorithm, analysis of the systematic error requires removal of stochastic sources of uncertainty, which in many cases can be very resource intensive.

Particle-based Monte Carlo techniques have found widespread use in a wide range of applications such as semiconductors (e.g. [33]), small-scale dilute-gas hydrodynamics (see the article [1]) and small-scale solid-state heat transfer, where their ability to efficiently treat high-dimensional problems offsets the limitations due to the statistical uncertainty associated with their results. The latter feature, namely the statistical fluctuations, can be an advantage or a disadvantage, depending on the application. 
In situations where accurate moment fields are required and the simulated phenomenon results in a small signal, statistical fluctuations become an "anathema" due to the large - sometimes prohibitive - computational cost associated with reducing the statistical uncertainty in the moment fields. This is the subject of the article by Al-Mohssen and Hadjiconstantinou [1] which demonstrates how the variance-reduction method of control variates can be used to reduce the statistical uncertainty associated with hydrodynamic field estimation in Monte Carlo particle-solution methods for the Boltzmann equation.

On the other hand, in fields where fluctuations are an important part of the physical model, the ability of the method to reproduce the correct fluctuation spectra is very important. This is the subject of the contribution by Bell et al. [3], which discusses the challenges associated with incorporating the correct hydrodynamic fluctuation spectra within traditional deterministic Navier-Stokes solution methods.

Although mean field particle methods have their foundations in numerical physics, and more specifically in fluid mechanics, recently they have found applications in a variety of engineering science fields as well as information theory. In fact, as computations have become more widespread, they have became a revolutionary stochastic simulation technology for solving complex estimation and optimization problems, including nonlinear filtering and data assimilation, rare event analysis, environmental studies, parameter estimation problems in hidden Markov chain models, stochastic control and optimal stopping problems.

These stochastic techniques are sometimes called sequential Monte Carlo methods in Bayesian statistics, and particle or bootstrap filters in advanced signal processing. In all the application domains discussed above, particle methods are used to approximate a flow of probability measures with an increasing level of complexity. This class of models includes conditional distributions of signal type states w.r.t. noisy and partial observations, non absorption probabilities in Feynman-Kac-Schrödinger models, Boltzmann-Gibbs measures with increasing inverse temperature concentrations, and conditional distributions of a stochastic process evolving in critical regimes. The time evolution equation of these distribution flows is governed by a nonlinear integro-differential equation for continuous time models, and by an updating-prediction type nonlinear dynamical system in distribution space for discrete generation models. For some rather regular classes of Feynman-Kac type particle models, several theoretical convergence results are available, including non asymptotic variance estimates, fluctuation theorems, exponential concentration inequalities, and large deviation theorems. Beside these theoretical recent advances, the mathematical foundations and the numerical analysis of these stochastic particle simulation models still remains a great challenge. We hope this special volume will facilitate the further development of this field and influence new application areas.

Stochastic particle algorithms belong to the class of Monte Carlo sampling techniques, and they offer a complementary tool and an alternative to standard deterministic function-based projections and deterministic grid-based algorithms, often restricted to low dimensional spaces and linear evolution models. Nevertheless, it is important to underline that stochastic methods are far from being reduced to Monte Carlo simulation algorithms. Several recent probabilistic type projection techniques are presented in this volume, including quantization grid methods, Malliavin calculus regression techniques, cubature methods, Wiener Chaos type expansions, and many others. These important and timely topics are not covered in depth in this special issue but are nevertheless included to provide better perspective on the field as a whole. The following papers serve this role, and hence round out our volume.

The article by Crisan and Manolarakis [8] provides an excellent survey on the applications of these advanced stochastic projection techniques to approximate semilinear partial differential equations arising in mathematical finance (with very detailed bibliographical notes). The article by Lototsky et al. [25] also discusses analytical and numerical approximations of elliptic equations by means of the Wiener Chaos (Cameron-Martin) expansions.

To guide the reader through this special volume on probabilistic interpretations of some classes of nonlinear integro-differential equations, and to make articles more accessible to researchers from different communities, we have tried below to provide a synthetic picture of the mathematical foundations and the range of applications of stochastic particle models. We have adopted an informal style and presentation, focusing on the ideas rather than on their rigorous mathematical justification. In each topic, we discuss in more details the contributions of each authors as well as the connections between some parts of their work. To provide a rather constructive and 
self contained volume, we have also added some selected references to classic works on stochastic processes and to more recent advances.

The rest of this introduction is organized as follows:

Section 2 is concerned with some more or less well known probabilistic interpretations of some partial differential equations, including diffusion processes and Fokker-Planck equations, jump type processes and bounded integral generators, as well as Euler type time discretization of diffusion and stochastic jump processes.

Section 3 is concerned with probabilistic interpretations of a class of nonlinear integro-differential equations, including parabolic type and McKean-Vlasov type diffusions, nonlinear jump models, collision type processes, as well as continuous or discrete time Feynman-Kac-Schrödinger semigroups. We provide a rather detailed presentation of the regularization techniques developed in three articles in the present volume, as well as a discussion on probabilistic interpretations of Dirichlet problems with boundary conditions and Poisson equations.

In Section 4, we provide an introduction to discrete and continuous time mean field particle model. We design a general and abstract class of stochastic interacting particle models and their probabilistic interpretations.

An introduction to the stochastic analysis of particle models is offered in the last section, Section 5, with special attention to the long time behavior and the numerical performance of these stochastic algorithms.

\section{Probabilistic interpretations of some Partial DifFEREntial EQUations}

\subsection{Diffusion processes and Fokker-Planck type equations}

It is instructive to briefly recall the well known probabilistic interpretation of the Fokker-Planck partial differential equation, also known as the Kolmogorov forward equation. Consider the $\mathbb{R}^{d}$-valued Itô stochastic differential equation

$$
\mathrm{d} X_{t}=b\left(X_{t}\right) \mathrm{d} t+\sigma\left(X_{t}\right) \mathrm{d} W_{t}
$$

with some initial random variable $X_{0}$ with distribution $\eta_{0}=\operatorname{Law}\left(X_{0}\right)$, where $W_{t}$ is a standard $d$-dimensional Wiener process, and for any $x \in \mathbb{R}^{d}, \sigma(x)=\left(\sigma_{i, j}(x)\right)_{1 \leq i, j \leq d}$ and $b(x)=\left(b_{i}(x)\right)_{1 \leq i \leq d}$ are respectively a symmetric nonnegative definite matrix and a vector. The first use of this equation was for the statistical description of Brownian motion of a particle in a fluid. Nowadays, these probabilistic models are very popular in a variety of application domains. The articles is this special volume illustrate the rich mathematical structure of these probabilistic models and their use in several selected applications including signal processing, information theory, bayesian statistics, fluid mechanics, molecular dynamics, as well as mathematical finance.

For instance, in [2] they are used to model the location of a fluid element through an acquisition path in turbulent fluid filtering problems, while in [4], they coincide with the Lagrangian fluid models that describe turbulent flows resulting from the particles' evolution. In a different context, in [8] these models are used to describe the complex stochastic evolution of assets and wealth portfolio processes in financial markets. In [16], these diffusion equations are rather used to model stochastic signal-observation evolutions in nonlinear filtering problems, or some reference Markov process in Feynman-Kac-Schrödinger semigroups. Another important application of these stochastic models are the Langevin dynamics defined by

$$
\mathrm{d} X_{t}=-\nabla V\left(X_{t}\right) \mathrm{d} t+\sqrt{2 \beta^{-1}} \mathrm{~d} W_{t} .
$$

Under some regularity conditions on the potential function $V$, the above diffusion is ergodic with an invariant measure given by the Boltzmann-Gibbs distribution $\left(\mathrm{e}^{-\beta V(y)} \mathrm{d} y\right)^{-1} \mathrm{e}^{-\beta V(x)} \mathrm{d} x$, sometimes referred to as the canonical measure. Since they concentrate on the global minima of the potential function $V$, these models are often used in stochastic optimization, as well as in physics to model the dynamics of molecular systems. Langevin dynamics and some of their variations are discussed in [21], as well as in [37].

Finally, we emphasize that these models also arise in a natural way in all the stochastic particle sampling methods discussed in this volume. In a sense, most of the stochastic models discussed in the series of articles dedicated to particle methods fit into this probabilistic framework. These comments will become clearer below, where mean field particle approximation models are discussed. 
We now discuss the connection between these probabilistic models and partial differential equations. Roughly speaking, using a second order Taylor's type expansion, for any infinitely differentiable test function $f$ of compact support, we have

$$
\mathrm{d} f\left(X_{t}\right)=L(f)\left(X_{t}\right) \mathrm{d} t+\mathrm{d} M_{t}(f)
$$

with the martingale remainder term

$$
M_{t}(f)=\sum_{1 \leq i, j \leq d} \int_{0}^{t} \partial_{i} f\left(X_{s}\right) \sigma_{i, j}\left(X_{s}\right) \mathrm{d} W_{s}^{j}
$$

and the second order-differential operator defined by

$$
L(f):=\frac{1}{2} \sum_{i, j=1}^{d} a_{i, j} \partial_{i, j}(f)+\sum_{1 \leq i \leq d} b_{i} \partial_{i}(f)
$$

with $a=\sigma^{2}$. This Taylor type second order expansion is known as the Itô's formula. For further details on this subject, we refer the reader to any textbook on stochastic calculus.

The magnitude of the stochastic "remainder" martingale term $M_{t}(f)$ given above is often measured in terms of its predictable angle bracket $\langle M(f)\rangle_{t}$. We recall that this predictable process is such that $M_{t}(f)^{2}-\langle M(f)\rangle_{t}$ is again a martingale, and it is defined below

$$
\langle M(f)\rangle_{t}=\int_{0}^{t} \Gamma_{L}(f, f)\left(X_{s}\right) \mathrm{d} s
$$

in terms of the "carré du champ" operator associated with $L$ and defined below

$$
\Gamma_{L}(f, f)(x)=L\left([f-L(f)(x)]^{2}\right)(x)=L\left(f^{2}\right)(x)-2 f(x) L(f)(x) .
$$

This stochastic analysis technique works for a fairly general class of infinitesimal generators $L$, including the jump type models discussed in the further development. For a more thorough discussion on these probabilistic models, we refer the reader to the seminal books by Revuz and Yor [35], Karatzas and Shreve [23] and Ethier and Kurtz [19].

The infinitesimal generator $L$ plays a fundamental role in the stochastic analysis of Markov processes and parabolic type partial differential equations. To be more precise, we let $\eta_{t}$ be the law of the random state $X_{t}$, and for any sufficiently regular function $g$ from $\mathbb{R}^{d}$ into $\mathbb{R}$, we set

$$
\eta_{t}(g):=\mathbb{E}\left(g\left(X_{t}\right)\right)=\int_{\mathbb{R}^{d}} \eta_{t}(\mathrm{~d} x) g(x)
$$

From (2.3), we readily find that

$$
\frac{\mathrm{d}}{\mathrm{d} t} \eta_{t}(f)=\eta_{t}(L(f))
$$

Furthermore, when the diffusion $X_{t}$ admits a density $p_{t}(x)$ w.r.t. the Lebesgue measure (which holds if the pair of functions $(a, b)$ are bounded and locally Lipschitz, and $a$ globally Hölder and uniformly positive definite), then we find that the above equation reads

$$
\left(\forall f, \quad \int f(x) \frac{\mathrm{d} p_{t}}{\mathrm{~d} t}(x) \mathrm{d} x=\int L(f)(x) p_{t}(x) \mathrm{d} x=\int f(x) L^{\star}\left(p_{t}\right)(x) \mathrm{d} x\right) \Longleftrightarrow \frac{\mathrm{d} p_{t}}{\mathrm{~d} t}=L^{\star}\left(p_{t}\right)
$$


with the adjoint operator $L^{\star}$ of $L$ obtained by a simple integration by parts, and defined below

$$
L^{\star}\left(p_{t}\right):=\frac{1}{2} \sum_{i, j=1}^{d} \partial_{i, j}\left(a_{i, j} p_{t}\right)-\sum_{1 \leq i \leq d} \partial_{i}\left(b_{i} p_{t}\right) .
$$

The partial differential equation in the r.h.s. of (2.6) is also known as the Fokker-Planck equation. From the pure probabilistic point of view, given an operator $L$, the existence of a Markov process with such an infinitesimal generator $L$ is only guaranteed by solving a martingale problem. In other words, we need to check that the Taylor type equation (2.3) is valid on a canonical space, for a sufficiently large class of test functions to ensure the uniqueness of the reference probability measure of the canonical Markov process. For a more thorough discussion on these martingale problems, we refer the reader to [19].

This technique is essential for ensuring that the continuous time stochastic model at hand is well posed on some probability space. In [21] the authors use this technique to check the existence and the uniqueness of a class of nonlinear diffusion models. It is also used in [7] to find a probabilistic interpretation of a family of divergence form operators with discontinuous coefficients at the interface of two open subsets of $\mathbb{R}^{d}$. This family of operators includes the case of the linearized Poisson-Boltzmann equation used to compute the electrostatic free energy of a molecule. The Markov processes discussed in this article strongly differ from (2.1) in the sense that the standard Wiener process is combined with non standard weighted local time terms at the boundaries of the interface of discontinuity. Using these Markov processes the authors design a novel probabilistic interpretation of divergence-form infinitesimal generators of the following form

$$
L=\nabla \cdot(\epsilon \nabla)
$$

where $\epsilon$ is a piecewise constant function from $\mathbb{R}^{d}$ into $\mathbb{R}$, with a smooth discontinuity manifold. For an introduction to these stochastic processes, we refer the reader to [34]. The extended version of Itô's formula to stochastic processes involving such local times relies on sophisticated probabilistic tools. In this contribution, the authors also provide a powerful generalized Itô-Meyer formula that also applies to non smooth functions.

\subsection{Jump type processes and integro-differential equations}

Of course all of the above probabilistic interpretation models are far from being restricted to second orderdifferential operators. For instance, let us replace in (2.5) the generator $L$ by the integro-differential operator $L+L^{\prime}$ with the bounded generator $L^{\prime}$ defined below

$$
L^{\prime}(f)(x)=V(x) \int(f(y)-f(x)) M(x, \mathrm{~d} y) .
$$

In the above display, $V$ stands for some bounded non negative function over $\mathbb{R}^{d}$ and $M(x, \mathrm{~d} y)$ a Markov transition from $\mathbb{R}^{d}$ into itself. When the Markov transition $M(x, \mathrm{~d} y)=m(x, y) \mathrm{d} y$ admits a density w.r.t. the Lebesgue measure, as in (2.6), we use Fubini's theorem to rewrite the jump's component of the weak sense equation (2.5) as follows

$$
\eta_{t}\left(L^{\prime}(f)\right)=\int\left\{\left[\int p_{t}(y) V(y) m(y, x) \mathrm{d} y\right]-p_{t}(x) V(x)\right\} f(x) \mathrm{d} x:=\int Q\left(p_{t}\right)(x) f(x) \mathrm{d} x .
$$

In this situation, we find that

$$
\frac{\mathrm{d} p_{t}}{\mathrm{~d} t}=L^{\star}\left(p_{t}\right)+Q\left(p_{t}\right)(x) \quad \text { with } \quad Q\left(p_{t}\right)(x):=\int p_{t}(y) V(y)\left[m(y, x) \mathrm{d} y-\delta_{x}(\mathrm{~d} y)\right] .
$$

The main advantage of these probabilistic formulations comes from the fact that the probability measures $\eta_{t}=\operatorname{Law}\left(X_{t}\right)$ can be interpreted as the law of a jump type Markov process $X_{t}$ that evolves between jumps 
times $T_{n}$ as in (2.1). The jump times $T_{n}$ are defined in terms of a sequence $\left(e_{n}\right)_{n \geq 1}$ of independent and identically exponentially distributed random variables with unit parameter by the following recursion

$$
T_{n}=\inf \left\{t \geq T_{n-1}: \int_{T_{n-1}}^{t} V\left(X_{s}\right) \mathrm{d} s \geq e_{n}\right\}
$$

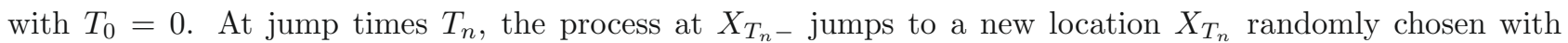
distribution $M\left(X_{T_{n}-}, \mathrm{d} y\right)$. For a more precise construction of these stochastic models, we refer the reader to [19]. The article by Muscato et al. [33] also provides a rather detailed discussion on these jump type probabilistic interpretations in the context of the Boltzmann transport equation.

By the law of large numbers, the law of the random states $\eta_{t}$ can be approximated by the occupation measure $\eta_{t}^{N}:=\frac{1}{N} \sum_{1 \leq i \leq N} \delta_{X_{t}^{i}}$ associated with $N$ independent copies $\left(X_{t}^{i}\right)_{1 \leq i \leq N}$ of a given stochastic process $X_{t}$, as $N \rightarrow \infty$, in the sense that

$$
\eta_{t}^{N}(f):=\frac{1}{N} \sum_{1 \leq i \leq N} f\left(X_{t}^{i}\right) \longrightarrow_{N \rightarrow \infty} \eta_{t}(f)=\mathbb{E}\left(f\left(X_{t}\right)\right)
$$

The above convergence estimate can be refined in various ways. Several asymptotic and non asymptotic estimates are available, including fluctuation theorems, exponential concentration inequalities, large deviation properties, as well as empirical process convergence analysis. For further details on this subject, we refer the reader to [41] or [17].

Besides the fact that these continuous time probabilistic models are directly connected to a rather large class of integro-differential equations, to get some computationally feasible solution, some kind of time discretization scheme is needed. In view of the stochastic differential equation (2.1), when mimicking traditional time discretization techniques of deterministic dynamical systems several natural strategies can be used. For instance, we can use an Euler type discretization of the diffusion (2.1)

$$
X_{t_{n}}^{\Delta}-X_{t_{n-1}}^{\Delta}=b\left(X_{t_{n-1}}^{\Delta}\right) \Delta+\sigma\left(X_{t_{n-1}}^{\Delta}\right)\left(W_{t_{n}}-W_{t_{n-1}}\right)
$$

on the time mesh $\left(t_{n}\right)_{n \geq 0}$, with $\left(t_{n}-t_{n-1}\right)=\Delta$, with some initial random variable with distribution $\eta_{0}=$ $\operatorname{Law}\left(X_{0}^{\Delta}\right)$. In this situation, the law $\eta_{n}$ of the approximated random states $X_{t_{n}}^{\Delta}$ satisfies the following linear integral equation

$$
\eta_{n}(\mathrm{~d} y)=\left(\eta_{n-1} K\right)(\mathrm{d} y):=\int \eta_{n-1}(\mathrm{~d} x) K(x, \mathrm{~d} y)
$$

with the elementary Markov transition

$$
K(x, \mathrm{~d} y):=\mathbb{P}\left(X_{t_{n}}^{\Delta} \in \mathrm{d} y \mid X_{t_{n-1}}^{\Delta}=x\right)
$$

This Gaussian transition can alternatively be defined in the integral form on bounded test functions as below

$$
K(f)(x):=\int K(x, \mathrm{~d} y) f(y)=\mathbb{E}(f(x+b(x) \Delta+\sigma(x) \sqrt{\Delta} V))
$$

where $V=\left(V^{i}\right)_{1 \leq i \leq d}$ is a sequence of independent and centered Gaussian random variables with unit variance. 
The jump type diffusion associated with the generator $L+L^{\prime}$ discussed above is defined as above by replacing the transition $K$ by the Markov transition

$$
(K S)(x, \mathrm{~d} z)=\int K(x, \mathrm{~d} y) S(y, \mathrm{~d} z) \quad \text { with } \quad S(y, \mathrm{~d} z)=\mathrm{e}^{-V(y) \Delta} \delta_{y}(\mathrm{~d} z)+\left(1-\mathrm{e}^{-V(y) \Delta}\right) M(y, \mathrm{~d} z)
$$

Notice that the stochastic exponential jump rate $V$ associated with the generator $L^{\prime}$ is approximated by a geometric time clock with parameter $\mathrm{e}^{-V \Delta}$. For further details on these approximation models, we refer the reader to any textbook on the discretization of stochastic differential equations. Non asymptotic and uniform variance estimates w.r.t. the time parameter for the Langevin dynamics equation (2.2) can be found in [39].

The accuracy of discrete time approximation models is also discussed in two contributions in the present volume: The article by Crisan and Manolarakis [8] provides a rather detailed discussion on time discretization techniques of forward backward stochastic differential equations. We also refer the reader to [33] for an alternative discrete time approximation model based on fictitious jump events. In this article, the authors also provide a discussion on discrete time approximation of the stochastic jump integrals introduced in the definition (2.8).

We emphasize that these discrete generation models can often be sampled easily using elementary simulation techniques of standard random variables. In the further development of this volume, this pair of additional approximation levels are implicitly assumed to be easily solved by some discrete time scheme and some numerical simulation tool. Finally, we mention that the martingale problems we discussed for continuous time stochastic models are easily solved for discrete generation models with a given elementary Markov transition $K$. In this situation the law $\mathbb{P}_{n}$ of any random path $\left(x_{0}, \ldots, x_{n}\right)$ is explicitly given by the formula

$$
\mathbb{P}_{n}\left(\mathrm{~d}\left(x_{0}, \ldots, x_{n}\right)\right)=\eta_{0}\left(\mathrm{~d} x_{0}\right) K\left(x_{0}, \mathrm{~d} x_{1}\right) \ldots K\left(x_{n-1}, \mathrm{~d} x_{n}\right)
$$

These constructions remain valid for general classes of discrete generation and time inhomogeneous stochastic processes on abstract measurable state spaces. The existence and the uniqueness of the law of "infinite" random path sequences $\left(x_{n}\right)_{n \geq 0}$ is proved by a projective limit argument and it is known as the Ionescu-Tulcea's extension theorem.

\section{Probabilistic interpretations of SOME NONLINEAR EQUATIONS}

\subsection{Diffusion models and parabolic type equations}

The extension of the above probabilistic interpretations to nonlinear partial differential equations is of course technically more involved, mainly because it requires the stochastic analysis of time inhomogeneous Markov processes whose evolutions depend on the distribution flow of their random states. Nevertheless, the central idea behind these sophisticated probabilistic models is rather simple. Next, we describe these rather elementary ideas, avoiding unnecessary stochastic analysis technicalities. We also present their application domain discussed in four contributions to this volume, including molecular dynamics, turbulent fluid modeling, and interacting particle evolution in diffusive matter.

Roughly speaking, suppose we are given a collection of infinitesimal generators $L_{t, \eta}$ indexed by the time parameter and the set of all probability measures $\eta$ over $\mathbb{R}^{d}$. These models have essentially the same form as the one given above, except that the parameter may depend on the time parameter $t$ or on probability measures $\eta$. Under appropriate regularity conditions, arguing as in (2.5), we consider the flow of probability measures $\eta_{t}$ defined for sufficiently regular functions by the following nonlinear equation

$$
\frac{\mathrm{d}}{\mathrm{d} t} \eta_{t}(f)=\eta_{t}\left(L_{t, \eta_{t}}(f)\right)
$$

The time inhomogeneous operators $L_{t, \eta_{t}}$ can be seen as the infinitesimal generators associated with a time inhomogeneous Markov process $\bar{X}_{t}$ whose evolution depends on the flow of distributions $\eta_{t}=\operatorname{Law}\left(\bar{X}_{t}\right)$. 
In the mean field particle model literature, this is sometimes referred to as the target process while equation (3.1) is referred to as the master equation.

To fix ideas, we can consider in (2.1) drift functions $b\left(., \eta_{t}\right)$ and diffusion terms $\sigma\left(\bar{X}_{t}, \eta_{t}\right)$ that depend on every time $t$ on the current distribution $\eta_{t}$ of the random state $\bar{X}_{t}$. In this situation, $\bar{X}_{t}$ is defined as

$$
\mathrm{d} \bar{X}_{t}=b\left(\bar{X}_{t}, \eta_{t}\right) \mathrm{d} t+\sigma\left(\bar{X}_{t}, \eta_{t}\right) \mathrm{d} W_{t} \quad \text { with } \quad \eta_{t}=\operatorname{Law}\left(\bar{X}_{t}\right)
$$

with some initial random variable $\bar{X}_{0}$ with distribution $\eta_{0}=\operatorname{Law}\left(\bar{X}_{0}\right)$.

Under appropriate regularity conditions, the derivation of the evolution equation (2.5) is still valid by replacing $L$ by the following collection of operators

$$
L_{t, \eta_{t}}(f):=\frac{1}{2} \sum_{i, j=1}^{d} a_{i, j}\left(., \eta_{t}\right) \partial_{i, j}(f)+\sum_{1 \leq i \leq d} b_{i}\left(., \eta_{t}\right) \partial_{i}(f) .
$$

In other words, for sufficiently regular test functions $f$ we have

$$
\mathrm{d} f\left(\bar{X}_{t}\right)=L_{t, \eta_{t}}(f)\left(\bar{X}_{t}\right) \mathrm{d} t+\mathrm{d} M_{t}(f) \quad \text { with } \quad\langle M(f)\rangle_{t}=\int_{0}^{t} \Gamma_{L_{t, \eta_{t}}}(f, f)\left(\bar{X}_{s}\right) \mathrm{d} s
$$

with some martingale remainder term $M_{t}(f)$. Under some appropriate regularity conditions, the diffusion $\bar{X}_{t}$ admits a density $p_{t}(x)$ w.r.t. the Lebesgue measure $\mathrm{d} x$; that is, we have that $\eta_{t}(\mathrm{~d} x)=p_{t}(x) \mathrm{d} x$. As in (2.6), by a simple integration by parts, we prove that

$$
\frac{\mathrm{d} p_{t}}{\mathrm{~d} t}:=\frac{1}{2} \sum_{i, j=1}^{\mathrm{d}} \partial_{i, j}\left(a_{i, j}\left(., \eta_{t}\right) p_{t}\right)-\sum_{1 \leq i \leq \mathrm{d}} \partial_{i}\left(b_{i}\left(., \eta_{t}\right) p_{t}\right) .
$$

This nonlinear partial differential equation has been introduced by McKean in his pioneering article on Propagation of chaos for a class of non-linear parabolic equations [30].

As for linear models, given a collection of operators $L_{t, \eta_{t}}$, the existence of a Markov process with such an infinitesimal time inhomogeneous generator is also guaranteed by solving a martingale type problem. In other words, we need to check that there exists a unique distribution on the space of random paths which allows one to consider in the stochastic process evolution a dependency on its finite time marginals. The solution to this problem is often obtained using a fixed point argument w.r.t. some transformation that associates to a given measure on path space the law of the stochastic process that evolves using the corresponding time marginals. The solution measure is called the McKean distribution. For a more thorough discussion of these measures, we refer the reader to the lecture notes of the Saint-Flour Probability Summer School in 1989 by Sznitman [38]. This technique is also used in [21].

These nonlinear diffusion equations are analyzed in several contributions of this volume. In the articles dedicated to turbulent fluid models, the stochastic process $\bar{X}_{t}=\left(\bar{X}_{t}^{1}, \bar{X}_{t}^{2}\right) \mathbb{R}^{d}=\mathbb{R}^{d_{1}} \times \mathbb{R}^{d_{2}}$ respectively describe the location and the velocity of a generic particle in a turbulent fluid. In [2,4], the drift function depends on the conditional distribution $P_{\eta_{t}}\left(x^{1} ; \mathrm{d} x^{2}\right)$ of the velocities $\bar{X}_{t}^{2}$, given the particle location $\bar{X}_{t}^{1}=x^{2}$, with respect to the current distribution $\eta_{t}$ of the fluid particle $\bar{X}_{t}$. If we set

$$
\eta_{t}^{1}:=\eta_{t} \circ\left(\bar{X}_{t}^{1}\right)^{-1}=\operatorname{Law}\left(\bar{X}_{t}^{1}\right)
$$

then, the conditional probability measure $P_{\eta_{t}}\left(x^{1} ; \mathrm{d} x^{2}\right)$ depends on the current measure $\eta_{t}$, and it is defined by the following equation

$$
\eta_{t}\left(\mathrm{~d}\left(x^{1}, x^{2}\right)\right)=\eta_{t}^{1}\left(\mathrm{~d} x^{1}\right) P_{\eta_{t}}\left(x^{1} ; \mathrm{d} x^{2}\right) .
$$


In the simplified Lagrangian stochastic model discussed by these authors the drift term $b^{1}\left(x^{1}, x^{2}, \eta\right)=x^{2}$, $\left(\sigma^{1, i}\right)_{i=1,2}$, are null, $\left(\sigma^{2, i}\right)_{i=1,2}$, do not depend on the current distribution and the velocities drift term is of the following form

$$
b^{2}\left(\left(x^{1}, x^{2}\right), \eta_{t}\right)=\mathbb{E}\left(B\left(\bar{X}_{t}\right) \mid \bar{X}_{t}^{1}=x^{1}\right)=\int P_{\eta_{t}}\left(x^{1} ; \mathrm{d} y^{2}\right) B\left(x^{1}, y^{2}\right)
$$

for some regular interaction kernel. More refined diffusion models of this type are discussed by these authors, including one or two dimensional isotropic flows with homogeneous turbulence, and three dimensional atmospheric Das and Durbin type flows with stratified turbulence.

In a different context, the Vlasov-Fokker-Planck equation discussed in [6] describes the evolution of the density of interacting and diffusive matter in the space of positions and velocities. In this situation, $\bar{X}_{t}=\left(\bar{X}_{t}^{1}, \bar{X}_{t}^{2}\right)$ represents again the location and the velocity of an elementary particle. This diffusive Newton's type equations are defined as above by choosing null diffusion functions $\sigma^{1, i}, i=1,2$, in the first coordinate, $\sigma^{2,1}=0$, the constant function $\sigma^{2,2}=\sqrt{2}$, and the drift terms

$$
b^{1}\left(\left(x^{1}, x^{2}\right), \eta_{t}\right)=x^{2} \quad \text { and } \quad b^{2}\left(\left(x^{1}, x^{2}\right), \eta_{t}\right)=-A\left(x^{2}\right)-B\left(x^{1}\right)-\int C\left(x^{1}-y^{1}\right) \eta_{t}\left(\mathrm{~d}\left(y^{1}, y^{2}\right)\right)
$$

The three terms $(A, B, C)$ model respectively the friction, the exterior confinement, and the interaction between particle in positions $x^{1}$ and $y^{1}$ in the underlying physical system.

Another important application of nonlinear diffusion in molecular dynamics simulation is discussed in [21]. The central idea is to outperform the convergence to equilibrium of the Langevin dynamics (2.2) using an adaptive biasing force diffusion model $\bar{X}_{t}$ that prevents the diffusion process from staying in metastable states by repelling the states where it stayed for too long a time. The mean force is again computed in terms of the conditional distribution of $\bar{X}_{t}$ w.r.t. to some judiciously chosen function that "flattens" the energy landscape in some direction. The nonlinear diffusion model associated with the first reaction coordinate corresponds the situation where $d_{1}=1$, and

$$
b\left(\left(x^{1}, x^{2}\right), \eta_{t}\right)=-\nabla V\left(x^{1}, x^{2}\right)+P_{\eta_{t}}\left(\partial_{1} V\right)\left(x^{1}\right) e_{1}
$$

where $e_{1}$ stands for the first vector in the canonical basis of $\mathbb{R}^{d}$. As in the Langevin dynamics model (2.2), the diffusion term $\sigma$ is constant and diagonal with $\sigma^{i, i}=\sqrt{2 \beta^{-1}}$.

Of course, all the nonlinear diffusion models depending on the conditional distributions $P_{\eta_{t}}$ are rather singular, mainly because these measures are generally unknown, and since they are not expressed in terms of integrals w.r.t. $\eta_{t}$, they are difficult to approximate. For instance, for diffusion models $\bar{X}_{t}=\left(\bar{X}_{t}^{1}, \bar{X}_{t}^{2}\right)$ with a probability density $p_{t}$ (w.r.t. the Lebesgue measure over $\mathbb{R}^{d_{1}+d_{2}}$ ), these conditional distributions have the following form

$$
P_{\eta_{t}}\left(x^{1} ; \mathrm{d} x^{2}\right)=\left(\int_{\mathbb{R}^{d_{2}}} p_{t}\left(x^{1}, x^{2}\right) \mathrm{d} x^{2}\right)^{-1} p_{t}\left(x^{1}, x^{2}\right) \mathrm{d} x^{2} .
$$

Using traditional kernel density estimation techniques, we can express $p_{t}$ in terms of an approximation model that depends on the current distribution $\eta_{t}$; that is, we have that

$$
p_{t}\left(x^{1}, x^{2}\right) \mathrm{d} x^{2} \simeq_{\delta \rightarrow 0} \int_{\mathbb{R}^{d_{1}}} g_{\delta}\left(x^{1}-y^{1}\right) p_{t}\left(y^{1}, x^{2}\right) \mathrm{d} y^{1} \mathrm{~d} x^{2}=\int_{\mathbb{R}^{d_{1}}} g_{\delta}\left(x^{1}-y^{1}\right) \eta_{t}\left(\mathrm{~d}\left(y^{1}, x^{2}\right)\right)
$$

for some smooth approximation $g_{\delta}(x)=\delta^{-d_{1}} g(x / \delta), \delta>0$, for the Dirac measure at the origin. The corresponding approximation of $P_{\eta_{t}}$ is defined below

$$
P_{\eta_{t}, \epsilon}^{\delta}\left(x^{1} ; \mathrm{d} x^{2}\right)=\left(\epsilon+\int_{\mathbb{R}^{d}} \eta_{t}\left(\mathrm{~d}\left(y^{1}, y^{2}\right)\right) g_{\delta}\left(x^{1}-y^{1}\right)\right)^{-1} \int g_{\delta}\left(x^{1}-y^{1}\right) \eta_{t}\left(\mathrm{~d}\left(y^{1}, x^{2}\right)\right)
$$


with some parameter $\epsilon \geq 0$. This regularization technique is used in four articles presented in this volume. In [4], the collection of functions $g_{\delta}$ are interpreted as mollifier functions, while in [2] they are seen as weak regularizing kernels; finally in [21], these techniques are rather interpreted in terms of the Nadaraya-Watson estimators of conditional expectations. In another context, the above kernel density estimation techniques are used in [1] to reconstruct the single-particle distribution function in a Boltzmann-type kinetic equation. We also refer the reader to [8] for a combination of these regularization techniques with the Malliavin calculus regression method.

\subsection{Interacting jump processes and nonlinear integro-differential equations}

Another important class of nonlinear equations (3.1) arising in physics and engineering sciences is concerned with integro-differential operators. For instance, let us replace in (2.5) the generator $L$ by the integro-differential operator $L+L_{\eta_{t}}^{\prime}$ with the bounded generator $L_{\eta_{t}}^{\prime}$ defined by

$$
L_{\eta_{t}}^{\prime}(f)(x)=V(x) \int(f(y)-f(x)) M_{\eta_{t}}(x, \mathrm{~d} y)
$$

In the above display, $M_{\eta}(x, \mathrm{~d} y)$ stands for a collection of Markov transition from $\mathbb{R}^{d}$ into itself indexed by the set of probability measures $\eta$ over $\mathbb{R}^{d}$. In this situation, equation (3.1) takes the form

$$
\frac{\mathrm{d}}{\mathrm{d} t} \eta_{t}(f)=\eta_{t}(L(f))+\eta_{t}\left(V M_{\eta_{t}}(f)\right)-\eta_{t}(V f)
$$

More complex Boltzmann-type kinetic models involving collision operators can also be written (in the weak sense) in terms of an interacting jump generator (in the velocity field) of the following form

$$
L_{\eta_{t}}^{\prime}(f)(x)=\int(f(z)-f(x)) \eta_{t}(\mathrm{~d} y) M_{\eta_{t}}((x, y), \mathrm{d} z)
$$

for some collision type integral operator $M_{\eta_{t}}((x, y), \mathrm{d} z)$. For homogeneous models $M_{\eta_{t}}((x, y), \mathrm{d} z)=M((x, y), \mathrm{d} z)$, the interacting jump term is given by the following quadratic collision term

$$
\eta_{t}\left(L_{\eta_{t}}^{\prime}(f)\right)=\int\left(\eta_{t} \otimes \eta_{t}\right)(\mathrm{d}(x, y)) M((x, y), \mathrm{d} z)[f(z)-f(x)]
$$

In all the situations discussed above, as for the diffusion models discussed in Section 3.1, the flow of measures $\eta_{t}=\operatorname{Law}\left(\bar{X}_{t}\right)$ can be interpreted as the distribution of the random states $\bar{X}_{t}$ of a time inhomogeneous jump type Markov process with an infinitesimal generator $L+L_{\eta_{t}}^{\prime}$ that depends on the current distribution $\eta_{t}$.

To illustrate these models, we examine the time evolution of the Markov process $\bar{X}_{t}$ associated with the infinitesimal generator $L+L_{\eta_{t}}^{\prime}$, with $L$ and $L_{\eta_{t}}^{\prime}$ given respectively in (2.4) and in (3.7). This Markov process is a jump-diffusion type process. The jump times $T_{n}$ are defined as in (2.8) by the following formula

$$
T_{n}=\inf \left\{t \geq T_{n-1}: \int_{T_{n-1}}^{t} V\left(\bar{X}_{s}\right) \mathrm{d} s \geq e_{n}\right\}
$$

with a sequence $\left(e_{n}\right)_{n \geq 1}$ of independent and identically exponentially distributed random variables with unit parameter. Between the jump times, the process $\bar{X}_{t}$ evolves according to the stochastic differential equation (2.1). At the $n$th jump time $T_{n}$, the random state $\bar{X}_{T_{n}-}$ jumps to a new location $\bar{X}_{T_{n}}$ randomly chosen with the distribution $M_{\eta_{T_{n}-}}\left(\bar{X}_{T_{n}-}, \mathrm{d} x\right)$, with the current distribution $\eta_{T_{n}-}=\operatorname{Law}\left(\bar{X}_{T_{n}-}\right)$ of the random state of the process before the jump. 
For a more precise description of these jump and collision type equations and their probabilistic interpretations in terms of generators, we refer the reader to the review article by Méléard [31], as well as the article by Tanaka [40].

A more physical and mechanical description of these Boltzmann kinetic models is provided in the present volume in [1] in the context of fluid mechanics and gaseous flows, as well as in [33] in the context of semiconductors. In the last referenced article, the stochastic particle evolution model represents the time-space evolution of electrons in the device. More precisely, the measure $\eta_{t}$ represents the probability distribution of the electron locations and the corresponding wave vectors. In this context, the "free motion" of particles is dictated by Newton's equations involving electron group velocities and electric field variations that also depends on the electron density in terms of the solution of a Poisson equation. Therefore, the free evolution generator $L$ as the one discussed in (3.8) is now replaced by an infinitesimal generator $L_{\eta_{t}}$ that also depends on the solution $\eta_{t}$. In this situation, the mean field stochastic particle model is also used in the free flight evolution to estimate at every time step the electric field in the electronic device.

We mention that Champagnat et al. also discuss probabilistic interpretations of the Poisson-Boltzmann equations describing the electrostatic potential around a biomolecular assembly [7].

\subsection{Feynman-Kac-Schrödinger distribution flows}

\subsubsection{Continuous time models}

In some particular instances the solution of (3.8) can be explicitly described by a functional Feynman-Kac type distribution. To describe these models, we examine the situation when the Markov transition reduces to $M_{\eta}(x, \mathrm{~d} y)=\eta(\mathrm{d} y)$, for any $x$. In this case, the above equation becomes quadratic

$$
\frac{\mathrm{d}}{\mathrm{d} t} \eta_{t}(f)=\eta_{t}(L(f))+\eta_{t}(V) \eta_{t}(f)-\eta_{t}(V f)
$$

and its solution is given by the following flow of Feynman-Kac type measures

$$
\eta_{t}(f)=\gamma_{t}(f) / \gamma_{t}(1) \quad \text { with } \quad \gamma_{t}(f):=\mathbb{E}\left(f\left(X_{t}\right) \exp \left\{-\int_{0}^{t} V\left(X_{s}\right) \mathrm{d} s\right\}\right)
$$

where $X_{t}$ stands for a Markov process with infinitesimal generator $L$, with some initial distribution $\gamma_{0}=\eta_{0}$. Using elementary computations, we also find that the flow of unnormalized measures $\gamma_{t}$ satisfies the following evolution equation

$$
\frac{\mathrm{d}}{\mathrm{d} t} \gamma_{t}(f)=\gamma_{t}\left(L^{V}(f)\right) \quad \text { with the Schrödinger operator } \quad L^{V}=L-V .
$$

The first rigorous derivation of this equation, known as the Feynman-Kac formula, in the context of Brownian motion evolving in an absorbing potential $V$ is due to Kac in 1949 [22].

Furthermore, using a simple derivation w.r.t. the time parameter of the logarithm of the normalizing constant $\gamma_{t}(1)$, we prove the following key result

$$
\gamma_{t}(1)=\mathbb{E}\left(\exp \left\{-\int_{0}^{t} V\left(X_{s}\right) \mathrm{d} s\right\}\right)=\exp \left\{-\int_{0}^{t} \eta_{s}(V) \mathrm{d} s\right\}
$$

from which we prove that the top eigenvalue $\lambda(V)$ of the Schrödinger operator $L^{V}$ (whenever it exists) is defined in terms of the long time average of the normalized Feynman-Kac flow; that is, we have that

$$
\lambda(V)=\lim _{t \rightarrow \infty} \frac{1}{t} \int_{0}^{t} \eta_{s}(V) \mathrm{d} s .
$$


We observe that $\eta_{t}$ are the marginal w.r.t. the time parameter $t$ of the Feynman-Kac distributions on the path space defined below

$$
\mathrm{d} \mathbb{Q}_{t}:=\frac{1}{\mathcal{Z}_{t}} \exp \left\{-\int_{0}^{t} V\left(X_{s}\right) \mathrm{d} s\right\} \mathrm{d} \mathbb{P}_{t} .
$$

In the above display, $\mathbb{P}_{t}$ stands for the distribution of the random paths $\left(X_{s}\right)_{0 \leq s \leq t}$, and $\mathcal{Z}_{t}=\gamma_{t}(1)$ is a normalizing constant.

These Feynman-Kac functional models play a central role in physics and engineering sciences, including non linear filtering, information theory, Bayesian inference, and mathematical finance. Five articles in this volume are more or less directly concerned with the stochastic analysis of these Feynman-Kac models in different application domains: Specially, we refer the reader to [8] on applications in mathematical finance, to [37] on the approximation of Dirichlet and "Fermion" ground state energies, to [2] on filtering problems of atmospheric fluid velocities along discrete acquisition systems and [16] on stochastic particle approximation of the full FeynmanKac path measures $\mathbb{Q}_{t}$, with applications in numerical physics and advanced signal processing, as well as in parameter estimation in hidden Markov chain problems. Finally, in [7] the reader will find some very nice extended Feynman-Kac formulae for linearized Poisson-Boltzmann equations arising in molecular dynamics.

Poisson type equations and related Dirichlet problems with boundary conditions arise in a variety of scientific disciplines, including in computational engineering, mathematical biology, fluid mechanics, as well as in molecular dynamics and statistical physics. The next section is devoted to some well known probabilistic interpretations of these equations.

\subsubsection{Dirichlet problems with boundary conditions}

In the traditional probabilistic interpretation, the Feynman-Kac measures (3.14) represent the distribution of the random paths of a particle evolving in an absorbing medium. The exponential weight $\exp \left\{-\int_{0}^{t} V\left(X_{s}\right) \mathrm{d} s\right\}$ is interpreted as the survival probability of a given random path $\left(X_{s}\right)_{0 \leq s \leq t}$. We let $T$ be the killing time of the process $X_{t}$ with state dependent rate $V$. We recall that this random time is defined as in (2.8) by setting $T_{0}=0$ and $T=T_{1}$. From the above discussion, for any bounded test function $f$, we have that

$$
Q_{t}(f)(x):=\mathbb{E}_{x}\left(f\left(X_{t}\right) 1_{t<T}\right)=\mathbb{E}_{x}\left(f\left(X_{t}\right) Z_{t}\right) \quad \text { with } \quad Z_{t}:=\exp \left\{-\int_{0}^{t} V\left(X_{s}\right) \mathrm{d} s\right\}
$$

where $\mathbb{E}_{x}($.$) stands for the expectation w.r.t. the distribution of the process X_{t}$ starting from $x$. Notice that $Q_{t}$ is the semigroup associated with the flow of measures $\gamma_{t}=\gamma_{s} Q_{t-s}, s \leq t$, defined in (3.11). The potential kernel or the Green's operator $\mathcal{G}$ associated with $T$ is given by

$$
\mathcal{G}(f)(x):=\mathbb{E}_{x}\left(\int_{0}^{T} f\left(X_{t}\right) \mathrm{d} t\right)=\int_{0}^{\infty} Q_{t}(f)(x) \mathrm{d} t=\mathbb{E}_{x}\left(\int_{0}^{\infty} f\left(X_{t}\right) Z_{t} \mathrm{~d} t\right)
$$

It is well known that this Green operator solves Poisson type equations associated with the Schrödinger operator $L^{V}$ defined in (3.11). More precisely, given a function $f$ and using (3.11) we prove that

$$
-L^{V}(g)=f \Rightarrow-Q_{t}(f)=Q_{t}\left(L^{V}(g)\right)=\frac{\mathrm{d}}{\mathrm{d} t} Q_{t}(g) \Rightarrow \mathcal{G}(f)=g
$$

as soon as $Q_{t}(g)$ tends to 0 , as $t \rightarrow \infty$. Thus, in this sense $\mathcal{G}=\left(-L^{V}\right)^{-1}$ is just the inverse of the Schrödinger operator. For general $V$, we have no chance of finding an explicit solution. For $V=0$, it is well known that $\mathcal{G}(f)(x)=-(2 \pi)^{-1} \int \log |x-y| f(y) \mathrm{d} y$ in $\mathbb{R}^{2}$, and $\mathcal{G}(f)(x)=(4 \pi)^{-1} \int(f(y) /|x-y|) \mathrm{d} y$ in $\mathbb{R}^{3}$.

Suppose that $-L^{V}(g)=f$ is only met on some bounded regular domain $D$ and we have $g=h$ on the boundary $\partial D$. We further assume that $f$ and $h$ are continuous on $D$ and $\partial D$, and $g$ is a bounded solution 
of the problem, with continuous first and second order derivatives in any interior sub-domain of $D$. Using (2.3) and a simple integration by part, we find that

$$
\mathrm{d}\left(g\left(X_{t}\right) Z_{t}\right)=Z_{t} L^{V}(g)\left(X_{t}\right) \mathrm{d} t+\mathrm{d} M_{t}^{Z}(g) \quad \text { with the martingale increment } \mathrm{d} M_{t}^{Z}(g):=Z_{t} \mathrm{~d} M_{t}(g) .
$$

Letting $T_{D}$ be the first exit time of $X_{t}$ from some domain $D$, we have

$$
g\left(X_{T_{D}}\right) Z_{T_{D}}=g(x)-\int_{0}^{T_{D}} f\left(X_{t}\right) Z_{t} \mathrm{~d} t+M_{T_{D}}^{Z}(g)
$$

so that the optional sampling theorem for martingales implies that

$$
g(x)=\mathbb{E}_{x}\left(g\left(X_{T_{D}}\right) Z_{T_{D}}+\int_{0}^{T_{D}} f\left(X_{t}\right) Z_{t} \mathrm{~d} t\right) .
$$

More general situations, including degenerate diffusion type generators and possibly unbounded domains are described in some detail in the book by Freidlin [20]. Discrete time versions and some particle approximations of these models can be also found in chapter 12 of the book by Del Moral [9].

In the present volume, one important contribution of the article by Champagnat et al. [7] is to provide new probabilistic interpretations of linearized Poisson-Boltzmann equations of the same form as above but for possibly unbounded domains, delta function sources, and replacing the diffusion type generator (2.4) by the divergence generator (2.7) with a piecewise constant and discontinuous diffusion term $\epsilon$. In this situation, the solution of the linearized Poisson-Boltzmann equation represents the electrostatic potential around a biomolecular assembly, composed of a finite number of atoms with fixed location and charges. The state space is decomposed into three parts, the interior of the molecule defined as the union of spheres representing the atoms, the exterior of the molecule and the interface manifold. The diffusion term $\epsilon$ represents the position-dependent dielectric, and it differs inside and out of the molecule. The absorption term $V$ is null inside the molecule, and it is a constant outside the molecule that reflects the ionic strength of the solution.

Combining the explicit formula of the electric field generated by a set of charges inside the molecule with a judicious regularization technique, the authors remove the delta function source terms and reduce the analysis to that of a regular Poisson equation on $\mathbb{R}^{3}$, with $C^{\infty}$-source terms with compact support inside the molecule. Thanks to this property, the complete solution of the problem now reduces to measuring the time average oscillations of the electric field at the interface during the evolution of the diffusion type process $X_{t}$, properly terminated during its visits outside the molecule.

Besides the fact that these mathematical results provide a significant contribution to the stochastic analysis of linearized Poisson-Boltzmann equations, the authors also provide a rigorous analysis, with detailed numerical comparisons and refined stochastic algorithms, of the Monte Carlo approximation method proposed by Mascagni and Simonov [29].

\subsubsection{Discrete time models}

Because of their importance in practice, we provide hereafter a brief presentation of the discrete time version of these Feynman-Kac models. These models are defined by the following formula

$$
\mathrm{d} \mathbb{Q}_{n}:=\frac{1}{\mathcal{Z}_{n}}\left\{\prod_{0 \leq p<n} G_{p}\left(X_{p}\right)\right\} \mathrm{d} \mathbb{P}_{n}
$$

where $\mathbb{P}_{n}$ stands for the distribution of the random paths $\left(X_{p}\right)_{0 \leq p \leq n}$ of a Markov chain, $G_{n}$ is a collection of $[0,1]$-valued potential functions, and $\mathcal{Z}_{n}$ another normalizing constant. As in the continuous time case, the weight factor $\left\{\prod_{0 \leq p<n} G_{p}\left(X_{p}\right)\right\}$ can be interpreted as the survival probability of a given random path $\left(X_{p}\right)_{0 \leq p<n}$, killed at rate $\left(1-G_{p}\right)$, at every time step $0 \leq p<n$. 
These discrete generation models arise in three articles in the present volume: In the article by Baehr, these models are used to describe the conditional distribution of a turbulent fluid w.r.t. atmospheric wind measurements along a sensor acquisition path. In this context, the potential functions represent both the likelihood function of a given wind observation as well as confinement type indicator functions associated with neighborhood balls along the sensor trajectories.

The law of the trajectories of the walk on sphere algorithm discussed in [7] can also be interpreted as a Feynman-Kac distribution of the form (3.15). In this situation, we have $X_{n}=\left(Y_{n}, R_{n}\right)$ where $Y_{n}$ is a random state in some given domain $D$ and $R_{n}$ the radius of the largest open sphere $S\left(Y_{n}, R_{n}\right)$ with center $Y_{n}$ and included in $D$. The Markov evolution $X_{n} \rightsquigarrow X_{n+1}$ consists in sampling randomly a point $Y_{n+1}$ in $\partial S\left(Y_{n}, R_{n}\right)$, and computing the corresponding new radius $R_{n+1}$. The survival probability of this model $G_{n}\left(X_{n}\right)=G_{n}\left(Y_{n}, R_{n}\right):=$ $R_{n} \sqrt{2 \lambda} / \sinh \left(R_{n} \sqrt{2 \lambda}\right)$ can be thought of as the survival probability of a Brownian particle starting from $Y_{n}$ when it hits $\partial S\left(Y_{n}, R_{n}\right)$, or equivalently $\lambda$ can be seen as the killing rate of the Brownian particle.

Other applications of these models in signal processing and hidden Markov chain models are also provided in [16]. In this contribution, the authors design a mean field particle approximation of integrals of additive functions $F_{n}\left(x_{0}, \ldots, x_{n}\right)=\frac{1}{n+1} \sum_{0 \leq p \leq n} f_{p}\left(x_{p}\right)$ w.r.t. the Feynman-Kac path measures $\mathbb{Q}_{n}$. These quantities arise in a natural way in the numerical solution of fixed parameter estimation problems in hidden Markov chain models. We shall return to this application at the end of this section.

These models can also be used to estimate the derivatives $\frac{\partial}{\partial \theta} \lambda\left(V^{\theta}\right)$ of ground state energies (3.13) associated with a collection of potential functions $V^{\theta}$ that depend smoothly on a parameter $\theta$. To describe with some precision these ideas, we recall that discrete time models are often deduced from their continuous time version by considering an additional Euler type discretization model with time step $\Delta$, and by setting $G_{n}\left(X_{n}\right):=\mathrm{e}^{-V\left(X_{n}\right) \Delta}$. The $\Delta$-approximation of the top eigenvalue $\lambda(V)$ defined in (3.13) is now given by the following quantities

$$
\lambda_{d}(G):=\lim _{n \rightarrow \infty} \lambda_{n, d}(G) \quad \text { with } \quad \lambda_{n, d}(G):=\frac{1}{n+1} \log \mathbb{E}\left(\prod_{0 \leq p \leq n} G\left(X_{p}\right)\right) .
$$

Considering a smooth collection of potential functions $\theta \mapsto G^{\theta}$, we readily check that the derivatives $\frac{\partial}{\partial \theta} \lambda_{n, d}\left(G^{\theta}\right)$ of the approximated ground state energies $\lambda_{n, d}\left(G^{\theta}\right)$ defined above are defined by the following additive functionals

$$
\frac{\partial}{\partial \theta} \lambda_{n, d}\left(G^{\theta}\right)=\mathbb{Q}_{n+1}^{\theta}\left(F_{n}^{\theta}\right) \quad \text { with } \quad F_{n}^{\theta}\left(x_{0}, \ldots, x_{n+1}\right):=\frac{1}{n+1} \sum_{0 \leq p \leq n} f^{\theta}\left(x_{p}\right) \quad \text { and } \quad f^{\theta}=\frac{\partial \log G^{\theta}}{\partial \theta}
$$

These functional derivatives of partition type functions and the sensitivity of normalizing constants of FeynmanKac measures that depends on a parameter $\theta$ arise in a number of application areas. Two rather different perspectives are discussed in this volume:

The article by Rousset [37] discusses the analog of these quantities for particle absorption models associated with a collection of hard obstacles depending sufficiently smoothly on a parameter $\theta$. In this context, the simple derivation techniques described above do not apply. To analyze this situation, the author provides a shape derivative formulation of the ground state energies using perturbation and variational techniques.

As we mentioned above, the article by Del Moral et al. [16] provides an application of these models in Bayesian inference and hidden Markov chain analysis. In this situation, the potential functions represent the likelihood functions of current observations, and the functional derivatives defined above represent the gradient of the log-likelihood of an observation sequence. In this context, these quantities are used to design efficient iterative procedures such as gradient ascent type algorithms. 


\section{An introduction to MEAN FIELd PARTICLE MODELS}

\subsection{Continuous time models}

The $N$-mean field particle interpretation of the evolution equation (3.1) is the Markov process $X_{t}^{(N)}=$ $\left(X_{t}^{(N, i)}\right)_{1 \leq i \leq N}$ on the product space $\left(\mathbb{R}^{d}\right)^{N}$ with generator $\mathcal{L}_{t}^{(N)}$ defined on sufficiently regular functions $F$ as follows

$$
\mathcal{L}_{t}^{(N)}(F)\left(x^{1}, \ldots, x^{N}\right):=\sum_{i=1}^{N} L_{t, \frac{1}{N} \sum_{i=1}^{N} \delta_{x^{i}}}^{(i)} F\left(x^{1}, \ldots, x^{i}, \ldots, x^{N}\right) .
$$

In the above displayed formula, we have used the upper index $L_{t, \eta}^{(i)}$ to indicate that the operator $L_{t, \eta}$ acts on the $i$ th coordinate. The initial system $X_{0}^{(N)}=\left(X_{0}^{(N, i)}\right)_{1 \leq i \leq N}$ consists of $N$ independent and identically distributed random variables with common law $\eta_{0}$.

In the situation discussed in (3.3), the $N$-mean field model is defined in terms of independent copies $\left(W_{t}^{i}\right)_{i \geq 1}$ of the Wiener process as follows

$$
\forall 1 \leq i \leq N \quad \mathrm{~d} X_{t}^{(N, i)}=b\left(X_{t}^{(N, i)}, \eta_{t}^{N}\right) \mathrm{d} t+\sigma\left(X_{t}^{(N, i)}, \eta_{t}^{N}\right) \mathrm{d} W_{t}^{i} \quad \text { with } \quad \eta_{t}^{N}:=\frac{1}{N} \sum_{i=1}^{N} \delta_{X_{t}^{(N, i)}} .
$$

This universal strategy is far from being restricted to diffusion type models. For instance, let us replace in (2.5) the generator $L$ by the integro-differential operator $L+L_{\eta_{t}}^{\prime}$ with the bounded generator $L_{\eta_{t}}^{\prime}$ defined in (3.7). As in (2.8), the $N$-mean field particle model associated with this class of generator is defined in terms of a jump-diffusion type Markov process $\left(X_{t}^{(N, i)}\right)_{1 \leq i \leq N}$ that evolves between jumps as independent copies of the diffusion (2.1). The jump times $T_{n}^{(N, i)}$ are defined as in (3.9) by the following formula

$$
T_{n}^{(N, i)}=\inf \left\{t \geq T_{n-1}^{(N, i)}: \int_{T_{n-1}^{(N, i)}}^{t} V\left(X_{s}^{(N, i)}\right) \mathrm{d} s \geq \mathrm{e}_{n}^{i}\right\}
$$

with a sequence $\left(\mathrm{e}_{n}^{i}\right)_{n, i \geq 1}$ of independent and identically exponentially distributed random variables with unit parameter. At jump times $T_{n}^{(N, i)}$, each particle $X_{T_{n}-(N, i)}^{(N, i)}$ jumps to a new location $X_{T_{n}^{(N, i)}}^{(N, i)}$ randomly chosen with distribution $M_{\eta_{T_{n-}^{N}}^{N}}\left(X_{T_{n}^{(N, i)}-}, \mathrm{d} y\right)$, with the current occupation measure $\eta_{T_{n}^{(N, i)}-}^{N}=\frac{1}{N} \sum_{i=1}^{N} \delta_{X_{T_{n}^{(N, i)}-}^{(N, i)}}$.

For the Feynman-Kac models discussed in (3.10), the time jumps $X_{T_{n}^{(N, i)}}^{(N, i)}$ are simply chosen randomly in the current population according to the distribution $\eta_{T_{n}^{(N, i)}}^{N}$. In this context, using formula (3.12) we define an unbiased particle approximation of the Feynman-Kac unnormalized distributions $\gamma_{t}$ by setting

$$
\gamma_{t}^{N}(f):=\eta_{t}^{N}(f) \times \exp \left\{-\int_{0}^{t} \eta_{s}^{N}(V) \mathrm{d} s\right\} \longrightarrow_{N \rightarrow \infty} \gamma_{t}(f):=\eta_{t}(f) \times \exp \left\{-\int_{0}^{t} \eta_{s}(V) \mathrm{d} s\right\} .
$$

The main advantage of these universal interacting stochastic sampling methods comes from the fact that the occupation measures $\eta_{t}^{N}:=\frac{1}{N} \sum_{i=1}^{N} \delta_{X_{t}^{(N, i)}}$ of the $N$-particle system satisfy the following equation

$$
\mathrm{d} \eta_{t}^{N}(f)=\eta_{t}^{N} L_{t, \eta_{t}^{N}}(f) \mathrm{d} t+\frac{1}{\sqrt{N}} \mathrm{~d} M_{t}^{N}(f)
$$

with a martingale process $M_{t}^{N}(f)$ such that

$$
\left\langle M^{N}(f)\right\rangle_{t}=\int_{0}^{t} \eta_{s}^{N} \Gamma_{L_{s, \eta_{s}^{N}}}(f, f) \mathrm{d} s
$$


As in (2.3), the proof of this result is a simple consequence of a "Taylor type" expansion that shows that for sufficiently regular test functions $F$ on the product space $\left(\mathbb{R}^{d}\right)^{N}$ we have that

$$
\mathrm{d} F\left(X_{t}^{(N)}\right)=\mathcal{L}_{t}^{(N)}(F)\left(X_{t}^{(N)}\right) \mathrm{d} t+\mathrm{d} \mathcal{M}_{t}^{(N)}(F) \quad \text { with } \quad\left\langle\mathcal{M}^{(N)}(F)\right\rangle_{t}=\int_{0}^{t} \Gamma_{\mathcal{L}_{s}^{(N)}}(F, F)\left(X_{s}^{(N)}\right) \mathrm{d} s
$$

If we choose empirical mean functions of the following form

$$
F\left(x^{1}, \ldots, x^{N}\right)=\frac{1}{N} \sum_{i=1}^{N} f\left(x^{i}\right)=\int m(x)(\mathrm{d} y) f(y) \quad \text { with the occupation measure } m(x):=\frac{1}{N} \sum_{i=1}^{N} \delta_{x^{i}}
$$

for any $x=\left(x^{1}, \ldots, x^{N}\right) \in\left(\mathbb{R}^{d}\right)^{N}$, then we conclude that

$$
L_{t, \frac{1}{N} \sum_{i=1}^{N} \delta_{x^{i}}}^{(i)}(F)(x)=\frac{1}{N} L_{t, m(x)}(f)\left(x^{i}\right) \Rightarrow \mathcal{L}_{t}^{(N)}(F)(x)=m(x)\left(L_{t, m(x)}(f)\right)
$$

In much the same way, we find that

$$
\begin{aligned}
\Gamma_{\mathcal{L}_{t}^{(N)}}(F, F)(x) & =\mathcal{L}_{t}^{(N)}\left(\left[F-\mathcal{L}_{t}^{(N)}(F)(x)\right]^{2}\right)(x) \\
& =\frac{1}{N^{2}} \sum_{i=1}^{N} \Gamma_{L_{t, m(x)}}(f, f)\left(x^{i}\right)=\frac{1}{N} m(x)\left(\Gamma_{L_{t, m(x)}}(f, f)\right)
\end{aligned}
$$

Notice that the particle profile evolution equation (4.1) only differs from the limiting model (3.1) by the Monte Carlo precision parameter $\frac{1}{\sqrt{N}}$ and the martingale fluctuation term $M_{t}^{N}(f):=\sqrt{N} \mathcal{M}_{t}^{(N)}(F)$.

From the above analysis, we can interpret mean-field particle models as a stochastic perturbation technique or as a kind of stochastic linearization type technique. From this perspective, to ensure that these stochastic perturbations do not propagate w.r.t. the time parameter, it is essential to study the robustness and the stability properties of the nonlinear limiting model (3.1). In other words, entering the stability properties of the semigroup of equation (3.1) in the performance analysis of a mean field particle approximation model, we expect to obtain useful uniform convergence estimates with respect to the time parameter. Of course, the study of the long time behavior of nonlinear evolution equations in distribution space of the form (3.1) is rather complex and it strongly depends on the model at hand. We shall return to this question in the last section of this introduction dedicated to the stochastic analysis of mean field particle models.

We end this section with a discussion on the statistical and physical interpretations of these probabilistic models. The mean field particle models presented above can be interpreted in different ways depending on the point of view of each particular application area; the list of different interpretations includes Stochastic adaptive grids in filtering problems and more general nonlinear integro-differential equations, microscopic or macroscopic descriptions of the evolution of physical observables, sophisticated Monte Carlo sampling technologies, branching and splitting schemes in rare event simulation, adaptive population evolutions in biology, and many others.

Most of these different interpretations can be found in the contributions of this volume: In [6], the stochastic particle model can be interpreted as a microscopic description of the evolution of an elementary diffusive particle interacting with matter. In $[2,4]$, the particle models provide a description of the evolution of an elementary fluid particle.

The continuous or discrete time generation Feynman-Kac type particle models discussed in [2,16,37], can be interpreted as a sophisticated interacting acceptance-rejection type Monte Carlo sampling technique, or as a natural stochastic and adaptive grid approximation. The particle models discussed in these three contributions are sometimes called sequential Monte Carlo methods in Bayesian inference and information theory, and evolutionary algorithms in stochastic optimization, while in numerical physics they are rather referred to 
as quantum or diffusion Monte Carlo methods. In this situation, the particles (also called walkers in physics) evolve according to some genetic type mutation and selection transitions.

In this connection, we mention that a common feature of these mean field particle models is that they allow one to compute, in a natural way, the normalizing constants of the Feynman-Kac measures introduced in (3.14) and (3.15). For continuous time models, the central idea is to replace in (3.12) the measures $\eta_{t}$ by their particle approximations $\eta_{t}^{N}$. In this way, one obtains an unbiased particle approximation of these quantities. These approximations are pivotal in the numerical solution of ground state energies in particle absorption models as well as in Bayesian statistics for computing likelihood functions. These two particle techniques are discussed in the articles referenced above.

The adaptive biasing force particle model presented in [21], can be interpreted as a new sophisticated and powerful Langevin type stochastic optimization technique for finding the global extrema of a potential energy function without getting trapped by the appearance of multiple metastable energy wells. The particle models discussed by these authors also represent the conformation of a protein taking into account the different microscopic and macroscopic time scales in the molecular simulation.

In the context of semiconductors, the stochastic particle model discussed in [33] mimics the evolution of electrons in time and space inside some device. Their behavior is determined by the action of external and self-consistent electric fields combined with stochastic scattering mechanisms.

Last but not least, in [1] the authors combine a microscopic particle-based simulation of a Boltzmann-type (non-equilibrium) gas model with judiciously chosen correlated data whose moments are known from equilibrium to achieve uncertainty reduction.

\subsection{Discrete time generation models}

As in the linear case discussed in the end of Section 2.2, to get some computationally feasible solution, an additional time discretization scheme is always needed. We recall that the solution $\eta_{t}$ of the nonlinear equation (3.1) can be interpreted as the law of the random states $\bar{X}_{t}$ of a nonhomogeneous Markov process whose evolution depends on the flow of measures $\eta_{t}$. Arguing as before, the law $\eta_{n}$ of the approximated random states $\bar{X}_{t_{n}}^{\Delta}$ on the time mesh $\left(t_{n}\right)_{n \geq 0}$, with $\left(t_{n}-t_{n-1}\right)=\Delta$, satisfies the following nonlinear integral equation

$$
\eta_{n}(\mathrm{~d} y)=\left(\eta_{n-1} K_{n, \eta_{n-1}}\right)(\mathrm{d} y):=\int \eta_{n-1}(\mathrm{~d} x) K_{n, \eta_{n-1}}(x, \mathrm{~d} y)
$$

with the Markov transition $K_{n, \eta_{n-1}}$ of a time inhomogeneous Markov process of the following form

$$
\mathbb{P}\left(\bar{X}_{t_{n}}^{\Delta} \in \mathrm{d} y \mid \bar{X}_{t_{n-1}}^{\Delta}=x\right)=K_{n, \eta_{n-1}}(x, \mathrm{~d} y) \quad \text { with } \quad \eta_{n-1}=\operatorname{Law}\left(\bar{X}_{t_{n-1}}^{\Delta}\right)
$$

with some initial random variable with distribution $\eta_{0}=\operatorname{Law}\left(\bar{X}_{0}^{\Delta}\right)$. In contrast with the continuous time diffusion type models discussed in Section 3.1, we mention that the McKean measure on random paths of length $n$ is now explicitly given by the following formula

$$
\mathbb{P}_{n}\left(\mathrm{~d}\left(x_{0}, \ldots, x_{n}\right)\right)=\eta_{0}\left(\mathrm{~d} x_{0}\right) K_{1, \eta_{0}}\left(x_{0}, \mathrm{~d} x_{1}\right) \ldots K_{n, \eta_{n-1}}\left(x_{n-1}, \mathrm{~d} x_{n}\right) .
$$

The $N$-mean field particle interpretation of the evolution equation (4.2) is now a Markov chain $X_{n}^{(N)}:=$ $\left(X_{n}^{(N, i)}\right)_{1 \leq i \leq N}$ on the product space $\left(\mathbb{R}^{d}\right)^{N}$ with elementary transitions given below

$$
\mathbb{P}\left(X_{n}^{(N)} \in \mathrm{d}\left(x^{1}, \ldots, x^{N}\right) \mid X_{n-1}^{(N)}\right)=\prod_{1 \leq i \leq N} K_{n, \eta_{n-1}^{N}}\left(X_{n-1}^{(N, i)}, \mathrm{d} x^{i}\right) \quad \text { with } \quad \eta_{n-1}^{N}:=\frac{1}{N} \sum_{i=1}^{N} \delta_{X_{n-1}^{(N, i)}} .
$$

The initial system $X_{0}^{(N)}=\left(X_{0}^{(N, i)}\right)_{1 \leq i \leq N}$ consists of $N$ independent and identically distributed random variables with common law $\eta_{0}$. 
The stochastic perturbation model associated with these discrete generation particle models is now defined in terms of a sequence of centered random fields $V_{n}^{N}$ defined by the following equation

$$
\eta_{n}^{N}=\eta_{n-1}^{N} K_{n, \eta_{n-1}^{N}}+\frac{1}{\sqrt{N}} V_{n}^{N}
$$

Once again, we notice that the above equation only differs from (4.2) by the Monte Carlo precision parameter $\frac{1}{\sqrt{N}}$ and the centered random fields $V_{n}^{N}$. Under rather weak regularity conditions, we can prove that $V_{n}^{N}$ converges in law, as $N \rightarrow \infty$, to a sequence of independent centered Gaussian fields $V_{n}$ with a variance function that can be explicitly expressed in terms of the Markov transitions $K_{n, \eta_{n-1}}$.

The stochastic perturbation analysis discussed above is developed in some details in the recent article by Del Moral and Rio [15]. For a more detailed discussion on Euler type approximations of McKean-Vlasov diffusion type models, we refer the reader to [28]. For a detailed analysis of Euler type approximations of Feynman-Kac type particle models in the context of molecular dynamics, we refer the reader to [18].

Most of the nonlinear integro-differential equations discussed in this volume can be approximated by a discrete generation model of the form given in (4.2). Discrete generation mean field particle models are always expressed as in (4.3), in terms of a Markov chain in a product space with elementary transitions that depend on the current occupation measure of the system. The limiting model (4.2) is simply obtained by replacing the occupation measures $\eta_{n}^{N}$ by the limiting measures $\eta_{n}$.

These discrete generation particle models are discussed in three articles in this volume: Article [2] discusses the discrete time confinement models as well as discrete time filtering problems of an elementary fluid particle along an acquisition path. In this situation, the mean field particle model combines an acceptance rejection transition with a particle genetic type filter. This article also provides a performance analysis of discrete generation and diffusion type mean field particle models. Article [33] is concerned with discrete evolution of electrons in a semiconductor. In this context, the authors discuss both the time discretization of Newton's equation of motions and the discrete time generation sampling of scattering times. This article also contains a thorough discussion with very nice numerical experiments on the way to combine these two discrete time approximation levels with mean field particle Monte Carlo sampling. Article [16] is dedicated to discrete time Feynman-Kac distribution flows. In this context, the Markov transition $K_{n, \eta}$ is the composition of a selection type mechanism and a free evolution transition. The corresponding mean field particle model can also be interpreted as a genetic type population evolution model.

Discrete generation models also provide a natural and easier way to design particle approximation of nonlinear equations and complex probability measures in path spaces, such as the Feynman-Kac functional models discussed in (3.14) and (3.15). Designing powerful particle approximations of these models is clearly much more involved than one of their finite time marginals. In the context of Feynman-Kac models, we recall that their interacting jump type and mean field particle approximations can be interpreted as a birth and death stochastic process combining free evolutions with spatial branching mechanisms.

In this context, two different particle approximations of models on path spaces can be underlined: The first one only consists of defining a mean field particle model in path space. From the pure mathematical point of view, up to a state space enlargement, this interpretation model coincides with the one of their finite time marginals. In this situation, path-valued particles can be interpreted as ancestral lines of the current individual population, and the occupation measure of the corresponding genealogical tree model converges to the desired distributions (3.14) and (3.15). In filtering problems, such as those discussed in [2,16], this genealogical tree based technique is sometimes used to compute the distribution of the full path of the signal given an observation sequence.

The second approximation model discussed in the last referenced article is based on a backward Markovian representation combined with the traditional mean field particle interpretation of the flow of their final time marginals. These particle models are rather based on the complete genealogical tree evolution associated with the mean field particle model. 


\section{Some Elements of Stochastic AnAlysis}

\subsection{Introduction}

The stochastic particle technology presented in this volume offers a unifying framework for the performance analysis of a variety of Monte Carlo type particle approximation models. For some rather regular classes of particle models, several theoretical convergence results are of course available, including propagation of chaos properties, fluctuation theorems, and large deviation principles. Providing an exhaustive review of this subject is clearly beyond the scope of this introduction. For McKean-Vlasov diffusion type models and Boltzmann collision type equations, we refer the reader to $[31,38]$.

The contributions of this volume dedicated to mean field particle models also contain a rather detailed and updated list of bibliographical references.

The present volume also provides several important contributions to this subject, with new regularity properties as well as original non asymptotic results for discrete or continuous time mean field particle models.

Article [21] provides a refined stochastic analysis of an adaptive biasing force type mean field particle model. The central difficulty comes from the fact that these stochastic particle models combine a mean field particle approximation with highly nonlinear conditional density estimations w.r.t. some reaction coordinate. A thorough discussion on the existence, the uniqueness and the regularity properties of the solutions of these nonlinear equation is presented, including the convergence of the regularized particle model. Article [4] provides a rigorous discussion on the existence and the uniqueness properties of nonlinear stochastic Lagrangian models of turbulent fluids. The authors also study the convergence of a regularized mean field particle model of the same form as the one discussed above. Article [2] is concerned with the stochastic analysis of mean field particle models arising in filtering problems of turbulent fluids. The authors provide new $\mathbb{L}_{p}$-mean error estimates for a rather general class of discrete generation particle models, including mean field type particle filtering models that depend on conditional distribution flows.

Beside these important theoretical advances, the numerical analysis and the long time behavior of these stochastic simulation models is still a great challenge nowadays. The main objective is to better calibrate their performance w.r.t. the size of the systems and other tuning parameters, including time discretization approximations, cooling decay rates, local random search strategies, interacting and adaptive search criteria, as well as population size parameters in branching type particle systems. One of the most important mathematical research tasks is to obtain uniform and non asymptotic precision estimates w.r.t. the time parameter so that to ensure a given performance index at any time horizon. Three contributions $[6,16,37]$ in this volume are concerned with these important questions.

A natural way to undertake this challenging task is to introduce the stability properties of the limiting nonlinear equations (3.1) in the analysis of the stochastic perturbation models discussed in (4.1) and (4.4). This strategy is rather standard in the numerical analysis of deterministic systems. For instance, it is often used to ensure that the local perturbation of a stable dynamical system does not propagate w.r.t. the time parameter. The development of these ideas in the context of mean field particle models seems to be more recent.

To the best of our knowledge, the first analysis of these stochastic perturbation models in the context of discrete generation particle models was developed in the article [10] and its extended version [11] published subsequently. The analysis of continuous time and interacting jump type particle models can be found in the series of articles [12-14]. For a more thorough discussion on the origins of this subject, with a rather detailed list of bibliographic references, we refer the readers to book [9].

For a more recent treatment, including sharp uniform rates w.r.t. the time parameter for continuous time Feynman-Kac models, we also refer the reader to article [36].

The application of these ideas to McKean-Vlasov diffusion type equations can be found in [26,27]. For a more recent treatment on this subject, we also refer the reader to article [5].

More recently, these ideas of entering the stability properties of the limiting semigroup into the propagation of chaos analysis have been used by Mischler and Mouhot [32] to derive strong uniform estimates w.r.t. the time parameter. 


\subsection{Stability properties of nonlinear evolution semigroups}

The stability analysis of Feynman-Kac type semigroups and Vlasov-Fokker-Planck models is discussed in three articles in the present volume. To describe these contributions in some detail, we provide a brief introduction to the Lipschitz type regularity properties of nonlinear semigroups $\Phi_{s, t}, s \leq t$, associated with a continuous time evolution equation (3.1), or discrete time dynamical systems of the form (4.2). We recall that these continuous or discrete time semigroups are defined for any sufficiently regular function $f$ by the following formula

$$
\Phi_{s, t}\left(\eta_{s}\right)(f):=\eta_{s}(f)+\int_{s}^{t} \eta_{r}\left(L_{r, \eta_{r}}(f)\right) \mathrm{d} r \quad \text { or by } \quad \Phi_{s, t}\left(\eta_{s}\right)=\eta_{s} K_{s+1, \eta_{s}} K_{s+2, \eta_{s+1}} \ldots K_{t, \eta_{t-1}} .
$$

In practice, the stability properties of these models are often described in terms of functional contraction inequalities. For instance in the context of discrete or continuous time Feynman-Kac models, under appropriate regularity conditions on the potential functions and the free evolution model, the following exponential asymptotic stability property is satisfied for any pair of probability measures $\eta, \eta^{\prime}$

$$
\left\|\Phi_{s, t}(\eta)-\Phi_{s, t}\left(\eta^{\prime}\right)\right\|_{\mathrm{tv}} \leq c \mathrm{e}^{-\lambda(t-s)}\left\|\eta-\eta^{\prime}\right\|_{\mathrm{tv}}
$$

for some finite constant $c<\infty$, and some exponential rate $\lambda>0$. In the above display, $\left\|\eta-\eta^{\prime}\right\|_{\text {tv }}$ stands for the total variation distance between probability measures. Several extensions of these inequalities to relative entropy like criteria, including Hellinger distances, $\mathbb{L}_{2}$-norms, and Boltzmann or Havrda-Charvat entropies can also be found in the book referenced above.

For time homogeneous models, these asymptotic stability results can be used to estimate the convergence of equilibrium of evolution equations of the form (3.10). These fixed measures $\eta_{\infty}$ are intimately related to the bottom eigenelements of the Schrödinger operator $L^{V}$ introduced in (3.11). For instance, using the long time average formula (3.13), it is readily verified that the largest eigenvalue $\lambda(V)$ of the Schrödinger operator $L^{V}$ (whenever it exists) as defined in (3.11) is given by $\lambda(V)=\eta_{\infty}(V)$. Furthermore, when the $L$-motion is reversible with respect to some reference distribution $\mu$, we have $d \eta_{\infty} \propto h d \mu$, with the eigenfunction $h$ associated with $\lambda(V)$. For further details on this subject, we refer the reader to the references given above as well as to the contribution of Rousset in the present volume [37] dedicated to hard obstacle absorption diffusion models and Dirichlet ground states energies.

Another discussion on the application of these stability results to discrete time Feynman-Kac models and the associated $h$-processes is provided in [16]. The authors also use these stability properties to control the oscillations of various semigroups involved in the study of the long time behavior of backward Markovian interpretations of Feynman-Kac measures on path spaces.

The contraction analysis of the Feynman-Kac models discussed above relies on specific semigroup techniques that do not apply to nonlinear diffusion type models of the form (3.2). The stability analysis of these diffusion models is often deduced using entropy techniques, log-Sobolev inequalities or $\mathbb{L}_{2}$-norms w.r.t. some equilibrium measure (whenever it exists), or using more traditional coupling techniques.

For instance, in the context of nonlinear Langevin diffusion type equations of the form discussed in (3.6), Lelièvre et al. [24] combine functional entropy inequalities with separation techniques of the microscopic and macroscopic scales to deduce the entropy of the $\mathbb{L}_{1}$-exponential convergence to the equilibrium. Roughly speaking, when the macroscopic term approaches equilibrium faster, the microscopic entropy follows an exponential decay to equilibrium associated with the stationary biasing force.

In the context of the nonlinear Vlasov-Fokker-Planck equations defined in (3.5), the equilibrium measure is also generally unknown so that the first two functional techniques discussed above do not apply. Notice that in this context, the fixed point distribution $\eta_{\infty}$ (whenever it exists) characterizes the limiting distribution of interacting and diffusive matter. In this situation, reference [6] in the present volume provides an original stability analysis on noncompact spaces using a simple and direct coupling method combined with powerful transportation inequalities w.r.t. the Wasserstein distance. These stochastic techniques fully exploit the probabilistic interpretation of the Vlasov-Fokker-Planck in terms of a time inhomogeneous diffusion process 
of the form (3.2). To be more precise, we recall that the Monge-Kantorovich or the Wasserstein distance $d$ of order 2 between two probability measures $\eta, \eta^{\prime}$ on $\mathbb{R}^{d}$ has the following optimal coupling characterization:

$$
d\left(\eta, \eta^{\prime}\right):=\inf _{X, X^{\prime}} \mathbb{E}\left(\left\|X-X^{\prime}\right\|^{2}\right)^{1 / 2}
$$

with the usual Euclidean distance $\left\|X-X^{\prime}\right\|$, and with an infimum taken over all random $\mathbb{R}^{d}$-valued random variables with marginal distributions $\eta=\operatorname{Law}(X)$, and $\eta^{\prime}=\operatorname{Law}\left(X^{\prime}\right)$.

The contraction properties developed by the authors provide an exponential convergence decay to equilibrium for Vlasov-Fokker-Planck diffusion models with quadratic-like friction and confinement forces, and sufficiently small Lipschitz interaction function. More precisely, if we let $\Phi_{s, t}\left(\eta_{s}\right)=\eta_{t}, s \leq t$, be the nonlinear semigroups associated with the Vlasov-Fokker-Planck equation, under the regularity properties discussed above, the authors prove the following contraction inequality

$$
d\left(\Phi_{s, t}(\eta), \Phi_{s, t}\left(\eta^{\prime}\right)\right) \leq c \mathrm{e}^{-\lambda(t-s)} d\left(\eta, \eta^{\prime}\right)
$$

for some finite constant $c<\infty$, and some exponential rate $\lambda>0$.

\subsection{Uniform convergence estimates}

Beside the fact that the contraction properties presented in the previous section provide some rather strong non asymptotic and exponential estimates on the stability properties of the limiting semigroups, the analysis of the long time behavior of mean field particle approximation models is technically much more involved. The reason is two-fold:

Firstly, mean field particle models are Markov chains in product state spaces with increasing dimensions. Therefore, the stability of the full population model often depends on the population size parameter. To by-pass these problems, we need to find a judicious way to reduce the analysis to their occupation measures. The second reason comes from the fact that most of the mean field particle models are not exact sampling methods, in the sense that the law of a given particle is not equal to the solution of the limiting equation. As a result, the stability property of the particle model cannot be directly deduced from the one of the limiting semigroup.

More formally, the central question is to find non asymptotic and uniform deviation estimates w.r.t. the time parameter for the couple of continuous time or discrete generation particle approximation models given below:

$$
\forall 1 \leq q \leq N \quad \mathbb{P}_{t}^{(N, q)}:=\operatorname{Law}\left(X_{t}^{(N, 1)}, \ldots, X_{t}^{(N, q)}\right) \longrightarrow_{N \rightarrow \infty} \eta_{t}^{\otimes q} \quad \text { and } \quad \eta_{t}^{N}:=\frac{1}{N} \sum_{i=1}^{N} \delta_{X_{t}^{(N, i)}} \longrightarrow N_{N \rightarrow \infty} \eta_{t} .
$$

In the l.h.s. convergence result $\eta_{t}^{\otimes q}$ stands for the $q$-tensor product of the limiting measure $\eta_{t}$. In other words, $\eta_{t}^{\otimes q}$ is the law of $q$ independent and identically distributed random sequences with common distribution $\eta_{t}$. By construction, at the origin we have $\mathbb{P}_{0}^{(N, q)}=\eta_{t}^{\otimes q}$. During their evolution, the particles interact with one another w.r.t. the current occupation measures $\eta_{t}^{N}$, whose values converge, as $N$ tends to infinity, to the deterministic limiting measures $\eta_{t}$. As a result, as $N$ tends to infinity, any finite block of particles asymptotically behaves as a collection of independent particles with the desired limiting distribution. In the literature of mean field particle models, this result is referred as the propagation of chaos property.

We emphasize that propagation of chaos properties are rather weak convergence properties, and as such, they only reflect the average independence degree and the bias of the particle approximation model. To illustrate this assertion, we quote the following more or less well known result which is valid for an indicator type function $F$ on $\left(\mathbb{R}^{d}\right)^{q}$, and for any particle model:

$$
\left|\mathbb{E}\left(\left(\eta_{t}^{N}\right)^{\otimes q}(F)\right)-\mathbb{P}_{t}^{(N, q)}(F)\right| \leq(q-1)^{2} / N
$$


In the context of discrete generation Feynman-Kac particle models, several uniform and strong propagation of chaos properties are available. For instance, using some rather strong stability properties on the limiting semigroup, we have the following estimate w.r.t. the total variation distance:

$$
\sup _{t \geq 0}\left\|\mathbb{P}_{t}^{(N, q)}-\eta_{t}^{\otimes q}\right\|_{\mathrm{tv}} \leq c(q) / N
$$

for some finite constant $c(q)<\infty$, whose values do not depend on the precision population size parameter $N$. Further details on these uniform inequalities, including various extensions to path space particle models, can be found in the series of articles on discrete or continuous time Feynman-Kac models presented in the end of Section 5.1.

The analysis of these interacting jump type particle models relies on specific semigroup and combinatorial techniques that do not apply to McKean-Vlasov diffusion type equations. In the space homogeneous case of granular media equation, Malrieu et al. provide a original bias analysis using log-Sobolev inequalities for the law of the $N$ particles at time $t$. In terms of the Wassertein distance defined in (5.1), this result takes the following form:

$$
\sup _{t \geq 0} d\left(\mathbb{P}_{t}^{(N, 1)}, \eta_{t}\right) \leq c(1) / \sqrt{N}
$$

For more details on this result, we refer the reader to the pair of articles by Malrieu referenced above. In the context of the Vlasov-Fokker-Planck equations defined in (3.5), the key Bakry-Emery curvature criterion used by Malrieu in the article referenced above does not apply, mainly because this diffusion model has a $-\infty$ curvature. To solve this problem, the authors in reference [6] in the present volume extend the above result combining Talagrand type transportation inequalities for the joint law of the particles and direct coupling techniques.

As we mentioned above, all of these bias type deviations are rather weak estimates. In practice, they are difficult to use to ensure a given precision index at any time horizon. To better calibrate the performance of a given stochastic particle model, it is preferable to analyze the $\mathbb{L}_{p}$-norm or the exponential deviation of the occupation measures $\eta_{t}^{N}$ of the particle model around their limiting value $\eta_{t}$.

Very few uniform results at the level of the empirical processes $f \mapsto \eta_{t}^{N}(f)$ are known. In the context of discrete generation Feynman-Kac model, for regular classes $\mathcal{F}$ of countable functions $f: \mathbb{R}^{d} \rightarrow \mathbb{R}$, with $\|f\| \leq 1$, and for any $p \geq 1$, we have the following uniform estimate

$$
\sup _{n \geq 0} \mathbb{E}\left[\sup _{f \in \mathcal{F}}\left|\eta_{t}^{N}(f)-\eta_{t}(f)\right|^{p}\right]^{1 / p} \leq c(p) / \sqrt{N}
$$

For instance, this inequality holds true for the set $\mathcal{F}:=\left\{1_{\left.\left.\prod_{i=1}^{d}\right] 0, x_{i}\right]}:\left(x_{i}\right)_{1 \leq i \leq d} \in \mathbb{R}^{d}\right\}$ of indicator of cells in $\mathbb{R}^{d}$. Working a little harder the estimation of the constants $c(p)$ in the above display, concentration exponential inequalities can be derived more or less directly from $\mathbb{L}_{p}$-mean error bounds. For instance, for a single test function $f$, with $\|f\| \leq 1$, we can prove the following uniform estimate w.r.t. the time parameter

$$
\left|\eta_{t}^{N}(f)-\eta_{t}(f)\right| \leq \epsilon+b_{1} / \sqrt{N} \quad \text { with a probability greater that } \quad 1-\mathrm{e}^{-N \epsilon^{2} / b_{2}}
$$

for any $\epsilon \geq 0$ and some finite constants $0 \leq b_{1}, b_{2}<\infty$. In the context of discrete generation Feynman-Kac models, these inequalities can be deduced from any $\mathbb{L}_{p}$-mean error bounds based on Kintchine's type inequalities with sharp constants w.r.t. the parameter $p$. In a recent article [15] the authors provide sharper bounds for general classes of discrete generation models, and replace the term $b_{1} / \sqrt{N}$ by $b_{1} / N$ and relate $\left(b_{1}, b_{2}\right)$ to the bias and the variance of the particle model.

All of the techniques discussed above do not apply to continuous time Feynman-Kac models, and their concentration properties still remain an important challenging problem.

In addition, at the level of trajectories, we do not expect uniform exponential concentration inequalities except for some particular classes of functions on path space. For Feynman-Kac discrete generation models 
on path space, the only known results in this direction are non uniform exponential concentration estimates with constants $\left(b_{1}, b_{2}\right)$ that are proportional to the current time horizon.

In the present volume, the authors of article [16] provide uniform estimates w.r.t. the time parameter for additive functionals of random paths under the Feynman-Kac distribution (3.14) and (3.15).

Article [6] provides a new strategy to obtain uniform concentration inequalities for regular Vlasov-FokkerPlanck particle models. The central idea is to control the deviation of the empirical measures $\eta_{t}^{N}(f)$ around their mean values $\mathbb{E}\left(\eta_{t}^{N}(f)\right)$ by proving a Talagrand type inequality for the joint law of the particles. This result combined with the bias estimates (5.2) gives an exponential concentration inequality of the form (5.3) for 1-Lipschitz functions.

Acknowledgements. The preparation of this special volume was a joint effort by a number of people. We are very grateful to the Chief Editors of the journal, C. Le Bris and A.T. Patera, who conceived and oversaw this project, and to Véronique Vacelet for her precious editorial assistance during the last two years.

Our special thanks also go to all the contributors to this special volume, as well as all the referees who further improved the submitted manuscripts by carefully reviewing the latter and providing useful comments and suggestions.

\section{REFERENCES}

[1] H.A. Al-Mohssen and N.G. Hadjiconstantinou, Low-variance direct Monte Carlo simulations using importance weights. ESAIM: M2AN 44 (2010) 1069-1083.

[2] C. Baehr, Nonlinear filtering for observations on a random vector field along a random vector field along a random path. Application to atmospheric turbulent velocities. ESAIM: M2AN 44 (2010) 921-945.

[3] J.B. Bell, A.L. Garcia and S.H. Williams, Computational fluctuating fluid dynamics. ESAIM: M2AN 44 (2010) $1085-1105$.

[4] F. Bernardin, M. Bossy, C. Chauvin, F. Jabir and A. Rousseau, Stochastic Lagrangian method for downscaling problems in meteorology. ESAIM: M2AN 44 (2010) 885-920.

[5] F. Bolley, A. Guillin and C. Villani, Quantitative concentration inequalities for empirical measures on non compact spaces. Prob. Theor. Relat. Fields 137 (2007) 541-593.

[6] F. Bolley, A. Guillin and F. Malrieu, Trend to equilibrium and particle approximation for a weakly selfconsistent VlasovFokker-Planck equation. ESAIM: M2AN 44 (2010) 867-884.

[7] N. Champagnat, M. Bossy and D. Talay, Probabilistic interpretation and random walk on spheres algorithms for the PoissonBoltzmann equation in molecular dynamics. ESAIM: M2AN 44 (2010) 997-1048.

[8] D. Crisan and K. Manolarakis, Probabilistic methods for semilinear PDEs. Application to finance. ESAIM: M2AN 44 (2010) $1107-1133$.

[9] P. Del Moral, Feynman-Kac formulae. Genealogical and interacting particle approximations, Series: Probability and Applications. Springer, New York (2004).

[10] P. Del Moral and A. Guionnet, On the stability of Measure Valued Processes with Applications to filtering. C. R. Acad. Sci. Paris, Sér. I 329 (1999) 429-434.

[11] P. Del Moral and A. Guionnet, On the stability of interacting processes with applications to filtering and genetic algorithms. Ann. Inst. Henri Poincaré 37 (2001) 155-194.

[12] P. Del Moral and L. Miclo, Branching and Interacting Particle Systems Approximations of Feynman-Kac Formulae with Applications to Non-Linear Filtering, in Séminaire de Probabilités XXXIV, J. Azéma, M. Emery, M. Ledoux and M. Yor Eds., Lecture Notes in Mathematics 1729, Springer-Verlag, Berlin (2000) 1-145.

[13] P. Del Moral and L. Miclo, Asymptotic stability of non linear semigroup of Feynman-Kac type. Ann. Fac. Sci. Toulouse Math. $11(2002)$ 135-175.

[14] P. Del Moral and L. Miclo, Particle approximations of Lyapunov exponents connected to Schrödinger operators and FeynmanKac semigroups. ESAIM: PS $\mathbf{7}$ (2003) 171-208.

[15] P. Del Moral and E. Rio, Concentration inequalities for mean field particle models. Ann. Appl. Probab. (to appear).

[16] P. Del Moral, A. Doucet and S.S. Singh, A backward particle interpretation of Feynman-Kac formulae. ESAIM: M2AN 44 (2010) 947-975.

[17] A. Dembo and O. Zeitouni, Large Deviations Techniques and Applications. Jones and Barlett Publishers, Boston (1993).

[18] M. El Makrini, B. Jourdain and T. Lelièvre, Diffusion Monte Carlo method: Numerical analysis in a simple case. ESAIM: M2AN 41 (2007) 189-213.

[19] S.N. Ethier and T.G. Kurtz, Markov processes: characterization and convergence, Wiley Series Probability ES Statistics. Wiley (1986).

[20] M. Freidlin, Functional integration and partial differential equations, Annals of Mathematics Studies 109. Princeton University Press (1985). 
[21] B. Jourdain, R. Roux and T. Lelièvre, Existence, uniqueness and convergence of a particle approximation for the adaptive biasing force. ESAIM: M2AN 44 (2010) 831-865.

[22] M. Kac, On distributions of certain Wiener functionals. Trans. Amer. Math. Soc. 65 (1949) 1-13.

[23] I. Karatzas and S.E. Shreve, Brownian Motion and Stochastic Calculus, Graduate Texts in Mathematics. Springer (2004).

[24] T. Lelièvre, M. Rousset and G. Stoltz, Long-time convergence of an adaptive biasing force method. Nonlinearity 21 (2008) $1155-1181$

[25] S. Lototsky, B. Rozovsky and X. Wan, Elliptic equations of higher stochastic order. ESAIM: M2AN 44 (2010) 1135-1153.

[26] F. Malrieu, Logarithmic Sobolev inequalities for some nonlinear PDE's. Stochastic Process. Appl. 95 (2001) $109-132$.

[27] F. Malrieu, Convergence to equilibrium for granular media equations and their Euler schemes. Ann. Appl. Probab. 13 (2003) $540-560$.

[28] F. Malrieu and D. Talay, Concentration inequalities for Euler schemes, in Monte Carlo and Quasi Monte Carlo Methods 2004, H. Niederreiter and D. Talay Eds., Springer (2005) 355-372.

[29] M. Mascagni and N.A. Simonov, Monte Carlo methods for calculating some physical properties of large molecules. SIAM J. Sci. Comput. 26 (2004) 339-357.

[30] H.P. McKean, Propagation of chaos for a class of non-linear parabolic equation, in Stochastic Differential Equations, Lecture Series in Differential Equations, Catholic Univ., Air Force Office Sci. Res., Arlington (1967) 41-57.

[31] S. Méléard, Asymptotic behaviour of some interacting particle systems; McKean-Vlasov and Boltzmann models, in Probabilistic Models for Nonlinear Partial Differential Equations 1627, Lecture Notes in Mathematics, Springer, Berlin-Heidelberg (1996) 44-95.

[32] S. Mischler and C. Mouhot, Quantitative uniform in time chaos propagation for Boltzmann collision processes. arXiv:1001. $2994 \mathrm{v} 1(2010)$.

[33] O. Muscato, W. Wagner and V. Di Stefano, Numerical study of the systematic error in Monte Carlo schemes for semiconductors. ESAIM: M2AN $4 \mathbf{4}$ (2010) 1049-1068.

[34] P. Protter, Stochastic integration and differential equations, Stochastic Modelling and Applied Probability 21. Springer-Verlag, Berlin (2005).

[35] D. Revuz and M. Yor, Continuous martingales and Brownian motion. Springer-Verlag, New York (1991).

[36] M. Rousset, On the control of an interacting particle approximation of Schrödinger ground states. SIAM J. Math. Anal. 38 (2006) 824-844.

[37] M. Rousset, On a probabilistic interpretation of shape derivatives of Dirichlet groundstates with application to Fermion nodes. ESAIM: M2AN 44 (2010) 977-995.

[38] A.-S. Sznitman, Topics in propagation of chaos, in Lecture Notes in Math 1464, Springer, Berlin (1991) $164-251$.

[39] D. Talay, Approximation of invariant measures on nonlinear Hamiltonian and dissipative stochastic different equations, in Progress in Stochastic Structural Dynamics 152, L.M.A.-C.N.R.S. (1999) 139-169.

[40] H. Tanaka, Stochastic differential equation corresponding to the spatially homogeneous Boltzmann equation of Maxwellian and non cut-off type. J. Fac. Sci. Univ. Tokyo, Sect. IA, Math. 34 (1987) 351-369.

[41] A.W. van der Vaart and J.A. Wellner, Weak Convergence and Empirical Processes. Second edition, Springer (2000). 